\title{
Étude par spectrométrie de masse de l'apparition des ions négatifs par attachement électronique dissociatif dans des molécules polyatomiques.
}

\author{
par R. LOCHT ${ }^{1}$ \\ Assistant à l'Université de Liège
}

\begin{abstract}
The appearance of negative ions by dissociative electron attachment has been studied by electron impact on $\mathrm{CH}_{4}$, $\mathrm{C}_{2} \mathrm{H}_{2}, \mathrm{C}_{2} \mathrm{H}_{4}$ and $\mathrm{C}_{2} \mathrm{H}_{3} \mathrm{X}(\mathrm{X}=\mathrm{F}, \mathrm{Cl})$ using a sectorfield mass spectrometer. An interpretation has been attempted for the measured appearance potentials. The electroaffinity of $\mathrm{C}_{2}$ and $\mathrm{C}_{2} \mathrm{H}$ radicals is found to be $(3,3 \pm 0,3) \mathrm{eV}$ and $(2,3 \pm 0,2) \mathrm{eV}$ respectively. Some evidence is brought for the existence of excited electronic states in polyatomic negative ions.
\end{abstract}

\section{INTRODUCTION}

Au cours des travaux antérieurs [1,2], nous avons exposé en détail les résultats d'une étude consacrée à l'apparition d'ions négatifs par impact électronique en atmosphère gazeuse raréfiée, et en particulier à l'apparition des ions négatifs par le processus d'attachement électronique dissociatif dans les molécules biatomiques.

Lors de l'attachement électronique dissociatif d'un électron à une molécule biatomique

$$
\mathrm{AB}+\mathrm{e}^{-} \rightarrow \mathrm{A}^{-}+\mathrm{B}
$$

la molécule subit une transition électronique vers le continuum d'un état électronique relatif au système $\mathrm{AB}^{-*}$. En admettant la validité du principe de Franck-Condon au cours de cette transition, il nous a été possible d'établir:

a) grâce au procédé de convolution, l'allure théorique de la courbe de variation de la section efficace $\sigma(\mathrm{E})$ d'attachement électronique dissociatif en fonction de l'énergie des électrons.

Cette courbe est étroitement liée à (i) la position relative des courbes d'énergie potentielle relatives à l'état initial $(\mathrm{AB})$ et à l'état final $\left(\mathrm{AB}^{*}\right)$ et (ii) à l'allure de la partie de la courbe d'énergie potentielle relative au système $\mathrm{AB}^{*}$ dans la zone de Franck-Condon. Ces deux éléments définissent la distribution d'énergie cinétique emportée par l'ion négatif.

b) une méthode non-arbitraire de détermination du seuil d'apparition des ions négatifs par attachement électronique dissociatif. Cette méthode consiste à prendre pour seuil d'apparition d'un ion négatif, le point d'intersection de l'extrapolation du flanc, situé vers les basses énergies, du pic correspondant avec l'axe d'énergie des électrons.

$\mathrm{Au}$ cours de ce travail, nous avons entrepris l'étude de l'apparition d'ions négatifs par attachement électronique dissociatif dans les molécules polyatomiques $\mathrm{CH}_{4}, \mathrm{C}_{2} \mathrm{H}_{2}, \mathrm{C}_{2} \mathrm{H}_{4}$ et $\mathrm{C}_{2} \mathrm{H}_{3} \mathrm{X}(\mathrm{X}=\mathrm{Cl}, \mathrm{F})$. Un travail antérieur, de Von Trepka [6], fut consacré à l'apparition d'ions négatifs par attachement électronique dissociatif dans $\mathrm{CH}_{4}, \mathrm{C}_{2} \mathrm{H}_{2}$ et $\mathrm{C}_{2} \mathrm{H}_{4}$. Nous avons repris l'étude de ces molécules dans le but de tenter d'interpréter le seuil de tous les processus observés. D'autres mécanismes, proposés par cet auteur, seront revus sur la base de données plus récentes d'énergies de dissociation des molécules et de chaleurs de formation des radicaux.

Pour déterminer le seuil des processus d'attachement électronique dissociatif, nous avons adopté la méthode d'extrapolation linéaire utilisée dans le cas des molécules biatomiques $[1,2]$.

\footnotetext{
${ }^{1}$ Présenté par M. L. D'OR.
} 


\section{CONDITIONS EXPERIMENTALES}

Les résultats expérimentaux qui seront mentionnés dans la suite de ce travail, ont été obtenus par impact d'électrons au moyen d'un spectromètre de masse du type Nier à secteur de $60^{\circ}$ (Atlas $\mathrm{CH} 4$ ) muni d'une source d'ions du type classique (AN4).

Les ions accélérés par un champ électrique constant de $3 \mathrm{Kv}$., sont analysés par un champ magnétique variable. Le courant ionique est détecté par un multiplicateur d'électrons du type H.E.T. à 17 dynodes dont la première dynode est à $2,3 \mathrm{Kv}$.

Le courant d'électrons, mesuré au piège, a été maintenu à $10 \mu \mathrm{A}$ et il a été vérifié que l'intensité du courant ionique est proportionnelle à la première puissance de la pression du gaz dans la chambre à ionisation (de l'ordre de $10^{-6}$ torr). Les courants électroniques et les pressions du gaz dans la source d'ions, généralement utilisés dans les travaux antérieurs [6], sont de $0,1 \mu \mathrm{A}$ à $3 \mu \mathrm{A}$ et de l'ordre de $10^{-4}$ torr respectivement.

Les conditions de focalisation ont été maintenues constantes de manière à obtenir une intensité maximale du pic d'attachement électronique dissociatif de l'ion $\mathrm{O}^{-}$dans $\mathrm{CO}$, dont le seuil a été choisi comme référence pour étalonner l'échelle d'énergie ; le seuil du processus

$$
\mathrm{CO}+\mathrm{e}^{-} \rightarrow \mathrm{O}^{-}+\mathrm{C}
$$

est situé à $9,6 \mathrm{eV}[3,4]$.

La valeur de chacun des potentiels d'apparition relatée dans ce travail résulte d'une moyenne de trois mesures indépendantes de la différence d'énergie entre le(s) seuil(s) caractérisant la courbe d'ionisation de l'ion étudié et le seuil de l'ion de référence, lequel a été enregistré pour chaque mesure. Toutes les mesures mentionnées ci-après sont suivies du plus grand écart à la moyenne. Un exemple de courbe d'ionisation est donné pour chaque ion.

\section{RÉSULTATS EXPÉRIMENTAUX ET LEUR DISCUSSION}

Avant de décrire les résultats expérimentaux et d'aborder leur discussion, rappelons les relations qui, pour un mécanisme proposé, et moyennant certaines hypothèses, permettront de calculer a priori le seuil d'un processus d'attachement électronique dissociatif.

Soit une molécule polyatomique $\mathrm{M}$ qui donne lieu au processus:

$$
\mathrm{M}+\mathrm{e}^{-} \rightarrow \mathrm{I}^{-}+\mathrm{R}
$$

Le potentiel d'apparition P.A. $\left(\mathrm{I}^{-}\right)$correspondant est donné par:

$$
\text { P.A. }\left(I^{-}\right)=D(I-R)-E A(I)+E_{c i n}+E_{e x c}
$$

où $\mathrm{D}(\mathrm{I}-\mathrm{R})$ est l'énergie de dissociation de la liaison $\mathrm{I}-\mathrm{R}, \mathrm{EA}(\mathrm{I})$ est l'électroaffinité de l'atome ou radical $\mathrm{I}, \mathrm{E}_{\mathrm{cin}}$ et $\mathrm{E}_{\mathrm{exc}}$ sont respectivement l'énergie cinétique et l'énergie d'excitation emportées par l'ion négatif et (ou) le(s) fragment(s) neutre(s).

A défaut de connaître le terme $\mathrm{D}(\mathrm{I}-\mathrm{R})$ et en admettant que $\mathrm{E}_{\mathrm{cin}}$ et $\mathrm{E}_{\mathrm{exc}}$ sont négligeables, l'évaluation a priori du seuil d'un processus d'attachement électronique dissociatif pourra être effectuée à partir de la relation:

$$
\text { P.A. }\left(\mathrm{I}^{-}\right)=\Delta \mathrm{U}_{\mathrm{f}}(\mathrm{I})+\Delta \mathrm{U}_{\mathrm{f}}(\mathrm{R})-\Delta \mathrm{U}_{\mathrm{f}}(\mathrm{M})
$$

où

$$
\Delta \mathrm{U}_{\mathrm{f}}\left(\mathrm{I}^{-}\right)=\Delta \mathrm{U}_{\mathrm{f}}(\mathrm{I})-\mathrm{EA}(\mathrm{I})
$$

et où $\Delta \mathrm{U}_{\mathrm{f}}(\mathrm{R}), \Delta \mathrm{U}_{\mathrm{f}}(\mathrm{M})$ et $\Delta \mathrm{U}_{\mathrm{f}}\left(\mathrm{I}^{-}\right)$sont les chaleurs de formation du (des) radical (radicaux) $\mathrm{R}$, de la molécule $\mathrm{M}$ et de l'ion négatif $\mathrm{I}^{-}$respectivement. Les valeurs adoptées pour les chaleurs de formation des radicaux et des molécules sont consignées en appendice. 


\section{A. LE METHANE $\mathrm{CH}_{4}$}

Dans le tableau I, nous avons mentionné les ions composant le spectre de masse des ions négatifs du méthane, les seuils d'apparition de ces ions ainsi que les résultats expérimentaux de Smith [5] et de Von Trepka [6]. Ce dernier utilise la méthode de l'extrapolation linéaire pour déterminer le seuil d'apparition des processus d'attachement électronique dissociatif.

L'ion $\mathrm{CH}_{3}{ }^{-}$est aussi observé; il est de très faible intensité et n'est pas mentionné ici.

TABLEAU I. Energie au seuil (en eV) des ions négatifs apparaissant dans $\mathrm{CH}_{4}$.

\begin{tabular}{|c|c|c|c|}
\hline & Ref. (5) & Ref. (6) & Ce travail \\
\hline $\mathrm{H}^{-}$ & 6,1 & & \\
\hline & 6,9 & $8,3 \pm 0,3$ & $8,2 \pm 0,2$ \\
\hline & 7,3 & $9,5 \pm 0,3$ & $9,4 \pm 0,15$ \\
\hline $\mathrm{CH}^{-}$ & 10,2 & $9,6 \pm 0,3$ & $9,7 \pm 0,2$ \\
\hline $\mathrm{CH}^{-}$ & 8,9 & $8,3 \pm 0,2$ & \\
\hline 2 & & $9,3 \pm 0,2$ & $9,3 \pm 0,3$ \\
\hline
\end{tabular}

\section{DISCUSSION}

a) $L^{\prime}$ ion $H^{\prime}$ (figure 1)

FIG. 1.

$\mathrm{H}^{-1} / \mathrm{CH}_{4}$

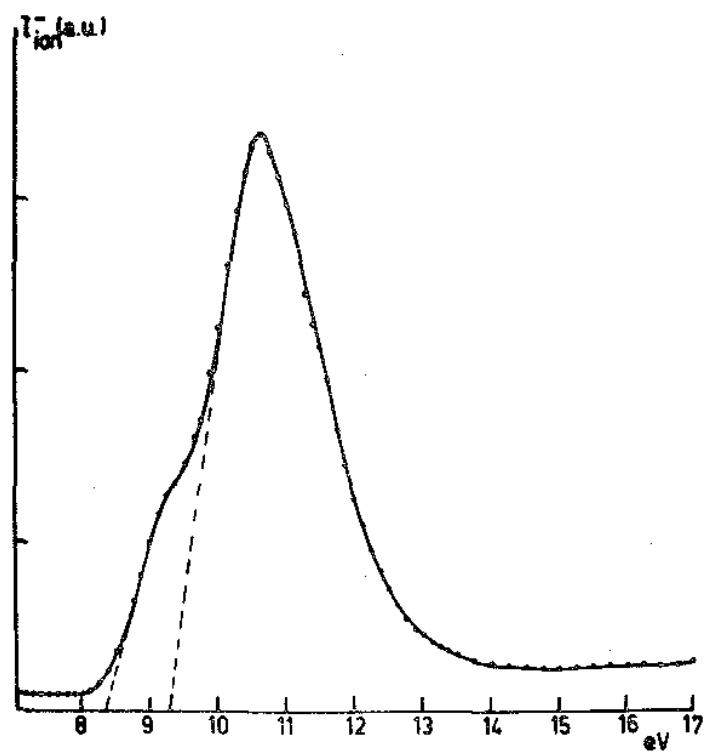

Dans le tableau II, nous avons consigné les mécanismes pouvant donner naissance à l'ion $\mathrm{H}^{-}$par le processus d'attachement électronique dissociatif à partir du méthane

Les potentiels d'apparition sont évalués au moyen de la relation (I) en adoptant les valeurs les plus récentes pour les énergies de dissociation $\mathrm{D}\left(\mathrm{CH}_{3}-\mathrm{H}\right)=4,41 \mathrm{eV}$ [7], $\mathrm{D}\left(\mathrm{CH}_{2}-\mathrm{H}\right)=4,90 \mathrm{eV}[8], \mathrm{D}(\mathrm{CH}-\mathrm{H})=4,23$ $\mathrm{eV}$ [8], $\mathrm{D}(\mathrm{C}-\mathrm{H})=3,47 \mathrm{eV}[9]$; pour l'électroaffinité $\mathrm{EA}(\mathrm{H})=0,75 \mathrm{eV}$ [10] et en admettant que $\mathrm{E}_{\mathrm{cin}}$ et $\mathrm{E}_{\text {exc }}$ sont nuls dans chaque cas. 


\section{TABLEAU II.}

\begin{tabular}{|c|c|c|}
\hline Mécanisme & P.A.(eV) & \\
\hline $\mathrm{CH}_{4}+\mathrm{e}^{-} \rightarrow \mathrm{CH}_{3}+\mathrm{H}^{-}$ & 3,66 & $(0)$ \\
\hline $\mathrm{CH}_{2}+\mathrm{H}+\mathrm{H}^{-}$ & 8,56 & $(2)$ \\
\hline $\mathrm{CH}+2 \mathrm{H}+\mathrm{H}^{-}$ & 12,76 & $(3 \mathrm{a})$ \\
\hline $\mathrm{CH}+\mathrm{H}_{2}+\mathrm{H}^{-}$ & 8,28 & $(3 \mathrm{~b})$ \\
\hline $\mathrm{C}+\mathrm{H}_{2}+\mathrm{H}+\mathrm{H}^{-}$ & 11,75 & $(4 \mathrm{a})$ \\
\hline $\mathrm{C}+3 \mathrm{H}+\mathrm{H}^{-}$ & 16,23 & $(4 \mathrm{~b})$ \\
\hline
\end{tabular}

Parmi ces mécanismes, seuls les deux premiers et $(3 b)$ peuvent retenir notre attention. L'expérience a montré [1] que les ions de faible nombre de masse sont affectés d'une forte discrimination d'énergie cinétique. Il a été montré que dans les conditions de focalisation des ions spécifiées plus haut, des ions $\mathrm{H}^{-}$emportant une énergie cinétique de l'ordre de $3 \mathrm{eV}$ ne sont pas collectés. L'énergie cinétique qu'emporterait l'ion $\mathrm{H}^{-}$, si le premier processus était en cause, serait de 4,6 eV. Le mécanisme le plus probable, donnant naissance à l'ion $\mathrm{H}^{-}$, serait

$$
\mathrm{CH}_{4}+\mathrm{e}^{-} \rightarrow \mathrm{CH}+\mathrm{H}_{2}+\mathrm{H}^{-}
$$

dont le potentiel d'apparition calculé $(8,28 \mathrm{eV})$ est en bon accord avec le seuil expérimental $(8,2 \pm 0,2) \mathrm{eV}$.

Le seuil observé à 9,4 eV peut être interprété par l'apparition de l'ion $\mathrm{H}^{-}$, soit (i) avec une faible quantité d'énergie cinétique et le radical $\mathrm{CH}$ et la molécule $\mathrm{H}_{2}$ apparaissant dans leur état vibronique fondamental, soit (ii) sans énergie cinétique et le radical $\mathrm{CH}$ ou la molécule $\mathrm{H}_{2}$ apparaissant dans un état vibrationnel excité.

\section{b) L'ion $\boldsymbol{C H}$ (figure 2)}

L'interprétation du processus d'attachement électronique dissociatif qui donne naissance à l'ion $\mathrm{CH}^{-}$ nécessite la connaissance de l'électroaffinité du radical $\mathrm{CH}$. Cette grandeur est mal connue expérimentalement [5, $6,11]$. Une valeur théorique, $\mathrm{EA}(\mathrm{CH})=1,6 \mathrm{eV}$, fut obtenue par Cade [12].

Les mécanismes permettant d'interpréter le seuil à 9,7 eV sont :

$$
\begin{aligned}
& \mathrm{CH}_{4}+\mathrm{e}^{-} \rightarrow \mathrm{CH}^{-}+\mathrm{H}_{2}+\mathrm{H} ; \mathrm{EA}(\mathrm{CH}) \geq-0,64 \mathrm{eV} \\
& \mathrm{CH}_{4}+\mathrm{e}^{-} \rightarrow \mathrm{CH}^{-}+3 \mathrm{H} ; \mathrm{EA}(\mathrm{CH}) \geq 3,74 \mathrm{eV}
\end{aligned}
$$

Du mécanisme (1), nous déduisons une valeur de l'électroaffinité du radical $\mathrm{EA}(\mathrm{CH}) \geq-0,64 \mathrm{eV}$, suivant que l'énergie cinétique emportée par l'ion est égale ou supérieure à zéro.

L'étude systématique de l'apparition de l'ion $\mathrm{CH}^{-}$à partir des molécules $\mathrm{C}_{2} \mathrm{H}_{2}$ et $\mathrm{C}_{2} \mathrm{H}_{4}$ par les processus d'attachement électronique dissociatif et de paire de paire d'ions ${ }^{2}$, nous a permis d'attribuer à $\mathrm{EA}(\mathrm{CH})$ la valeur $(2,6 \pm 0,3) \mathrm{eV}$ (voir ci-après et [13]).

Le premier mécanisme d'apparition de l'ion $\mathrm{CH}^{-}$paraît être le plus vraisemblable, mais où l'ion $\mathrm{CH}^{-}$ emporterait une certaine quantité d'énergie cinétique et (ou) de l'énergie d'excitation vibronique. La molécule d'hydrogène peut apparaître dans un état vibrationnel excité.

Lorsque nous examinons le schéma des états électroniques relatifs au radical $\mathrm{NH}$, isoélectronique de l'ion $\mathrm{CH}^{-}$, nous constatons que, dans un domaine de $4 \mathrm{eV}$, on observe l'existence de quatre états électroniques excités [9].

Si $\mathrm{X}^{3} \Sigma^{-}$est l'état fondamental de l'ion $\mathrm{CH}^{-}$, la valeur $\mathrm{EA}(\mathrm{CH})=1,6 \mathrm{eV}$, calculée par Cade, correspondrait à l'ion $\mathrm{CH}^{-}$dans le premier état excité et $\mathrm{EA}(\mathrm{CH})=-0,64 \mathrm{eV}$ correspondrait à l'apparition de l'ion $\mathrm{CH}^{-}$dans un deuxième état électronique excité ou dans le premier état vibronique excité en emportant une certaine quantité

\footnotetext{
${ }^{2} \mathrm{Au}$ cours du processus de formation d'une paire d'ions, la molécule subit une excitation vibronique suivie d'une dissociation en un ion négatif et un ion positif par le mécanisme: $\mathrm{AB}+\mathrm{e}^{-} \rightarrow \mathrm{AB}^{*}+\mathrm{e}^{-}$puis $\mathrm{AB}^{*} \rightarrow \mathrm{A}^{+}+\mathrm{B}^{-}$
} 
Published in: Bulletin de la Classe des Sciences. Académie Royale de Belgique (1970), vol. LVI, pp. $788-817$.

Status: Postprint (Author's Version)

d'énergie cinétique.

FIG. 2.



c) L'ion $\mathrm{CH}_{2}^{-}$(figure 3)

Les seuls mécanismes qui puissent donner naissance à l'ion $\mathrm{CH}_{2}{ }^{`}$ sont:

$$
\begin{aligned}
& \mathrm{CH}_{4}+\mathrm{e}^{-} \rightarrow \mathrm{CH}_{2}^{-}+\mathrm{H}_{2} \\
& \mathrm{CH}_{4}+\mathrm{e}^{-} \rightarrow \mathrm{CH}_{2}^{-}+2 \mathrm{H}
\end{aligned}
$$

FIG. 3.

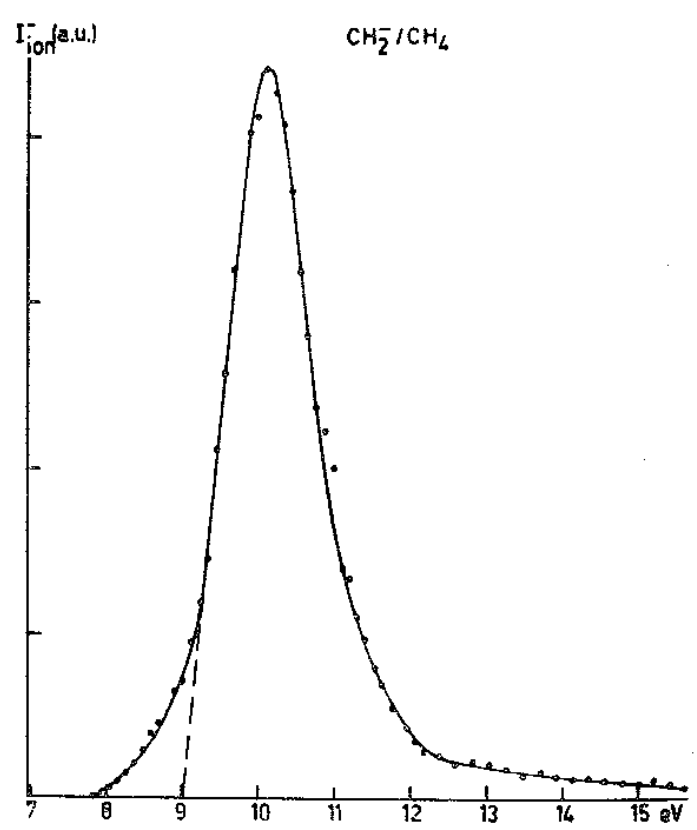


La valeur de l'électroaffinité du radical $\mathrm{CH}_{2}$, calculée à partir de ces deux mécanismes, est de $\mathrm{EA}_{1}\left(\mathrm{CH}_{2}\right)=$ $-4,48 \mathrm{eV}$ et $\mathrm{EA}_{2}\left(\mathrm{CH}_{2}\right)=0,0 \mathrm{eV}$ respectivement. Le mécanisme (2) interprète le mieux le seuil observé à $(9,3 \pm 0,3)$ $\mathrm{eV}$.

\section{B. L'ACETYLENE $\mathrm{C}_{2} \mathrm{H}_{2}$}

Dans le spectre de masse des ions négatifs de l'acétylène, seuls les ions $\mathrm{H}^{-}, \mathrm{C}_{2}$ et $\mathrm{C}_{2} \mathrm{H}^{-}$apparaissent par le processus d'attachement électronique dissociatif. Dans le tableau III, nous avons rassemblé les résultats expérimentaux obtenus au cours de ce travail et par Von Trepka [6],

TABLEAU III. Énergie au seuil des ions négatifs apparaissant dans $\mathrm{C}_{2} \mathrm{H}_{2}$.

\begin{tabular}{|c|c|c|}
\hline & Réf. (6) & Ce travail \\
\hline $\mathrm{H}^{-}$ & $7,6 \pm 0,3$ & $7,2 \pm 0,1$ \\
\hline $\mathrm{C}_{2}{ }^{-}$ & $7,6 \pm 0,2$ & $7,5 \pm 0,2$ \\
\hline & $11,8 \pm 0,3$ & $11,6 \pm 0,2$ \\
\hline & & $12,2 \pm 0,2$ \\
\hline $\mathrm{C}_{2} \mathrm{H}^{-}$ & $2,8 \pm 0,2$ & $2,8 \pm 0,2$ \\
\hline & $6,0 \pm 0,3$ & $7,5 \pm 0,2$ (max.) \\
\hline
\end{tabular}

\section{DISCUSSION}

a) L'ion $\boldsymbol{H}$ (figure 4)

FIG. 4.

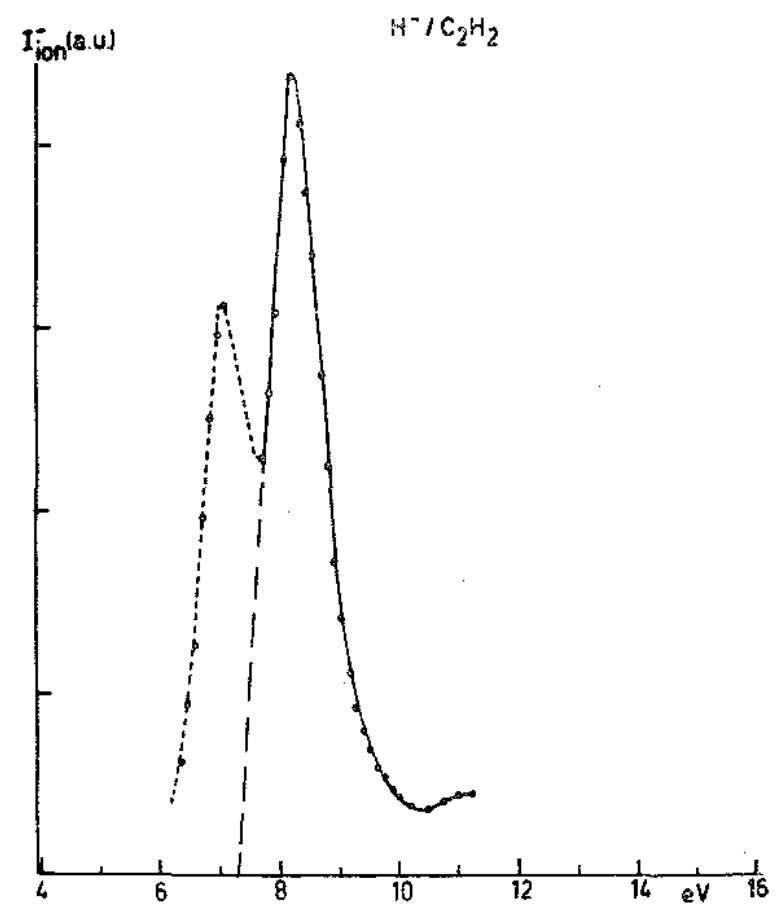

Le seuil du seul processus d'attachement électronique dissociatif observé, donnant naissance à $\mathrm{H}^{-}$, est situé à $7,2 \mathrm{eV}$; le pic qui naît vers $6 \mathrm{eV}$ est dû à la présence d'eau résiduelle dans la source d'ions. Le processus d'attachement électronique dissociatif qui donne naissance à l'ion $\mathrm{H}^{-}$à partir de $\mathrm{H}_{2} \mathrm{O}$ a une grande section efficace. Le processus de formation de l'ion $\mathrm{H}^{-}$dans $\mathrm{C}_{2} \mathrm{H}_{2}$ est de faible intensité. 
Au maximum des pics d'attachement électronique dissociatif relatifs à la formation de l'ion $\mathrm{H}^{-}$à partir de $\mathrm{H}_{2} \mathrm{O}, \mathrm{CH}_{4}$ et $\mathrm{C}_{2} \mathrm{H}_{2}$, enregistrés dans des conditions expérimentales identiques, l'intensité, exprimée en unités arbitraires, est de 350 u.a., 66 u.a. et 7 u.a. respectivement.

Dans le Tableau IV, nous avons consigné les mécanismes pouvant donner naissance à l'ion $\mathrm{H}^{-}$par attachement électronique dissociatif.

TABLEAU IV.

\begin{tabular}{|c|lc|}
\hline Mécanismes & \multicolumn{2}{|c|}{ P.A.calc. (eV) } \\
\hline $\mathrm{C}_{2} \mathrm{H}_{2}+\mathrm{e}^{-} \rightarrow \mathrm{C}_{2} \mathrm{H}+\mathrm{H}^{-}$ & 4,15 & $(1)$ \\
\hline $\mathrm{C}_{2}+\mathrm{H}+\mathrm{H}^{-}$ & 10,05 & $(2)$ \\
\hline $\mathrm{CH}+\mathrm{C}+\mathrm{H}^{-}$ & 12,60 & $(3)$ \\
\hline $2 \mathrm{C}+\mathrm{H}+\mathrm{H}^{-}$ & 16,07 \\
\hline
\end{tabular}

Seul le mécanisme (1) peut retenir notre attention. Pour la raison indiquée plus haut, la différence d'énergie de $3 \mathrm{eV}$ entre le seuil expérimental et le potentiel d'apparition calculé ne peut être attribuée à l'énergie cinétique emportée par l'ion $\mathrm{H}^{-}$.

Il est vraisemblable que l'ion $\mathrm{H}^{-}$emporte peu, ou pas, d'énergie cinétique et que le radical $\mathrm{C}_{2} \mathrm{H}$ apparaît dans un état vibronique excité.

$$
\mathrm{C}_{2} \mathrm{H}_{2}+\mathrm{e}^{-} \rightarrow \mathrm{C}_{2} \mathrm{H}^{*}+\mathrm{H}^{-}
$$

b) L'ion $\mathrm{C}_{2}^{-}$(figure 5)

FIG. 5.

$\mathrm{C}_{2}-1 \mathrm{C}_{2}+\mathrm{H}_{2}$

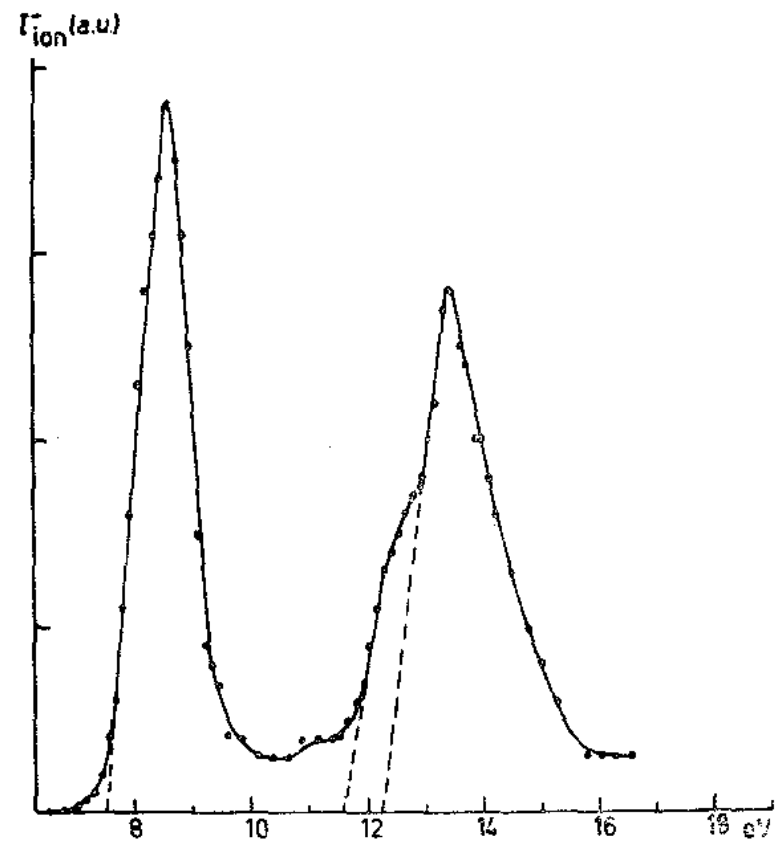

Trois pics correspondant à $\mathrm{C}_{2}^{-}$ont été observés à $7,5 \mathrm{eV}, 11,6 \mathrm{eV}$ et $12,2 \mathrm{eV}$ respectivement. Deux mécanismes peuvent donner naissance à l'ion $\mathrm{C}_{2}^{-}$par le processus d'attachement électronique dissociatif dans l'acétylène. 


$$
\begin{aligned}
& \mathrm{C}_{2} \mathrm{H}_{2}+\mathrm{e}^{-} \rightarrow \mathrm{C}_{2}^{-}+\mathrm{H}_{2} \\
& \mathrm{C}_{2} \mathrm{H}_{2}+\mathrm{e}^{-} \rightarrow \mathrm{C}_{2}^{-}+2 \mathrm{H}
\end{aligned}
$$

A partir de ces deux mécanismes et de la valeur du premier seuil, nous calculons la chaleur de formation de l'ion $\mathrm{C}_{2}^{-}$, soit (1) $\Delta \mathrm{U}_{\mathrm{f}}\left(\mathrm{C}_{2}^{-}\right)=9,85 \mathrm{eV}$ et (2) $\Delta \mathrm{U}_{\mathrm{f}}\left(\mathrm{C}_{2}^{-}\right)=5,33 \mathrm{eV}$, en admettant que l'ion $\mathrm{C}_{2}^{-}$apparaisse sans énergie cinétique. La valeur de l'électroaffinité du radical $\mathrm{C}_{2}$ serait de $-1,12 \mathrm{eV}$ et $3,3 \mathrm{eV}$ respectivement. Cette dernière valeur est en bon accord avec la valeur de 3,1 eV, la plus basse obtenue par Honing [14] par sublimation du graphite. Le mécanisme le plus probable pour l'interprétation du seuil observé à $7,5 \mathrm{eV}$, sera:

$$
\mathrm{C}_{2} \mathrm{H}_{2}+\mathrm{e}^{-} \rightarrow \mathrm{C}_{2}^{-}\left(\mathrm{X}^{2} \Sigma_{\mathrm{g}}{ }^{+}\right)+2 \mathrm{H}\left({ }^{2} \mathrm{~S}_{\mathrm{g}}\right)
$$

où l'ion $\mathrm{C}_{2}^{-}$apparaît à l'état fondamental $\mathrm{X}^{2} \Sigma_{\mathrm{g}}{ }^{+}$, par comparaison avec les molécules biatomiques isoélectroniques $\mathrm{CN}$ et $\mathrm{N}_{2}^{+}$.

Les seuils déterminés à $11,6 \mathrm{eV}$ et $12,2 \mathrm{eV}$ ne peuvent être attribués qu'à l'apparition de l'ion $\mathrm{C}_{2}^{-}$dans un état vibronique excité.

Les deux premiers états électroniques excités du radical $\mathrm{CN}$ sont $\mathrm{A}^{2} \Pi$ et $\mathrm{B}^{2} \Sigma^{+}$situés respectivement à $9241 \mathrm{~cm}^{-1}(1,15 \mathrm{eV})[9]$ et $25752 \mathrm{~cm}^{-1}(3,19 \mathrm{eV})$ [9].

Il est vraisemblable qu'à $11,6 \mathrm{eV}$ et $12,2 \mathrm{eV}$ le mécanisme qui donne naissance à l'ion $\mathrm{C}_{2}{ }^{-}$soit

$$
\mathrm{C}_{2} \mathrm{H}_{2}+\mathrm{e}^{-} \rightarrow \mathrm{C}_{2}^{-}\left(\mathrm{B}^{2} \Sigma^{+}\right)+2 \mathrm{H}\left({ }^{2} \mathrm{~S}_{\mathrm{g}}\right)
$$

où l'ion $\mathrm{C}_{2}^{-}$apparaîtrait soit dans l'état $\mathrm{B}^{2} \Sigma^{+}$vibrationnellement excité, soit dans l'état $\mathrm{B}^{2} \Sigma^{+}$fondamental en emportant une certaine quantité d'énergie cinétique.

\section{z) L'ion $\mathrm{C}_{2} \mathrm{H}$ (figure 6).}

FIG. 6.

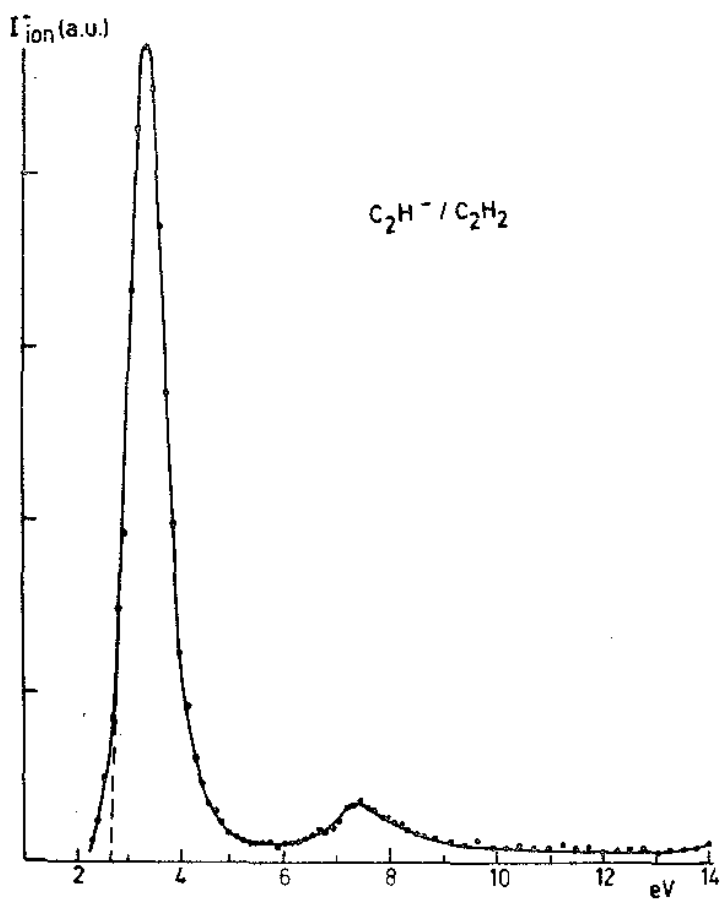

Le seul mécanisme permettant d'interpréter le processus observé à $2,8 \mathrm{eV}$ est 


$$
\mathrm{C}_{2} \mathrm{H}_{2}+\mathrm{e}^{-} \rightarrow \mathrm{C}_{2} \mathrm{H}^{-}+\mathrm{H}
$$

A partir de $\mathrm{D}\left(\mathrm{C}_{2} \mathrm{H}-\mathrm{H}\right)=4,90 \mathrm{eV}[8]$, nous obtenons $(2,1 \pm 0,2) \mathrm{eV}$ pour la valeur de l'électroaffinité du radical $\mathrm{C}_{2} \mathrm{H}$, l'ion $\mathrm{C}_{2} \mathrm{H}^{-}$emportant peu ou pas d'énergie cinétique.

Nous observons un deuxième processus de très faible intensité, caractérisé par un maximum à $7,5 \mathrm{eV}$ et dont le seuil est estimé à $6,6 \mathrm{eV}$. L'intervalle d'énergie de 3,8 eV est attribuable à l'excitation de l'ion $\mathrm{C}_{2} \mathrm{H}^{-}$. Dans la molécule de $\mathrm{HCN}$, isoélectronique de $\mathrm{C}_{2} \mathrm{H}^{-}$, le premier état électronique excité est situé à $6,48 \mathrm{eV}$ [8]. Le mécanisme le plus probable correspondant au seuil à $6,6 \mathrm{eV}$ serait:

$$
\mathrm{C}_{2} \mathrm{H}_{2}+\mathrm{e}^{-} \rightarrow \mathrm{C}_{2} \mathrm{H}^{-*}+\mathrm{H}\left({ }^{2} \mathrm{~S}_{\mathrm{g}}\right)
$$

\section{L'ÉTHYLÈNE $\mathbf{C}_{2} \mathrm{H}_{4}$}

Les résultats expérimentaux relatifs aux ions négatifs composant le spectre de masse de l'éthylène sont repris dans le tableau V: en parallèle, nous mentionnons les résultats expérimentaux obtenus par Von Trepka [6].

TABLEAU $\boldsymbol{V}$. Énergie de seuil (en eV) des ions négatifs apparaissant dans $\mathrm{C}_{2} \mathrm{H}_{4}$.

\begin{tabular}{|c|c|c|}
\hline & Réf. (6) & Ce travail \\
\hline $\mathrm{H}^{-}$ & $7,6 \pm 0,3$ & $7,5 \pm 0,3$ \\
\hline & $9,7 \pm 0,4$ & $9,6 \pm 0,1$ \\
\hline $\mathrm{CH}^{-}$ & $7,3 \pm 0,4$ & - \\
\hline & $8,7 \pm 0,3$ & $9,0 \pm 0,1$ \\
\hline $\mathrm{CH}_{2}^{-}$ & $8,8 \pm 0,3$ & - \\
\hline $\mathrm{C}_{2}^{-}$ & $10,4 \pm 0,3$ & - \\
\hline $\mathrm{C}_{2} \mathrm{H}^{-}$ & $7,1 \pm 0,4$ & $6,9 \pm 0,1$ \\
\hline & $10,0 \pm 0,3$ & $9,9 \pm 0,05$ \\
\hline
\end{tabular}

$\mathrm{L}^{\prime}$ ion $\mathrm{H}^{-}$est de très faible abondance et nous n'avons pas détecté de courants ioniques mesurables dus aux ions $\mathrm{CH}_{2}{ }^{-}$et $\mathrm{C}_{2}{ }^{-}$à basse énergie, dans le domaine de $5 \mathrm{eV}$ à $12 \mathrm{eV}$. Nous n'observons pour l'ion $\mathrm{C}_{2}^{-}$que l'apparition par processus de paire d'ions. Dans le domaine de pressions de $10^{-4}$ torr. Von Trepka [6] détecte ces ions à basse énergie, entre $7 \mathrm{eV}$ et $10 \mathrm{eV}$.

\section{DISCUSSION}

\section{a) L'ion $\boldsymbol{H}$ (figure 7)}

Le pic situé vers $6 \mathrm{eV}$ est dû à l'eau résiduelle dans la chambre à ionisation. Parmi les mécanismes de formation de l'ion $\mathrm{H}^{-}$par attachement électronique dissociatif nous ne mentionnerons dans le tableau VI que ceux dont le P.A.calc est égal ou inférieur à $\mathrm{PA}_{\mathrm{exp}}$.

\section{TABLEAU VI.}

\begin{tabular}{|c|cc|}
\hline Mécanismes & \multicolumn{2}{|c|}{ P.A.calc. (eV) } \\
\hline $\mathrm{C}_{2} \mathrm{H}_{4}+\mathrm{e}^{-}->\mathrm{C}_{2} \mathrm{H}_{3}+\mathrm{H}^{-}$ & 3,70 & (a) \\
\hline $\mathrm{C}_{2} \mathrm{H}_{4}+\mathrm{e}^{-} \rightarrow \mathrm{C}_{2} \mathrm{H}_{2}+\mathrm{H}+\mathrm{H}^{-}$ & 5,58 & (b) \\
\hline $\mathrm{C}_{2} \mathrm{H}_{4}+\mathrm{e}^{-} \rightarrow \mathrm{C}_{2} \mathrm{H}+\mathrm{H}_{2}+\mathrm{H}^{-}$ & 6,08 & (c) \\
\hline
\end{tabular}

Les différences d'énergie entre le premier seuil situé à 7,5 eV et les potentiels d'apparition calculés sont respectivement de $3,8 \mathrm{eV}, 2,0 \mathrm{eVet} 1,4 \mathrm{eV}$.

Ces différences d'énergie peuvent être attribuées: $1^{\circ}$ à l'énergie cinétique emportée par les fragments, essentiellement par $\mathrm{H}^{-}$ou $\mathrm{H}_{\text {et }} \mathrm{H}^{-}$, et l'énergie d'excitation vibronique des radicaux; pour les raisons mentionnées plus haut, seuls les mécanismes (b) et (c) peuvent être considérés; $2^{\circ}$ à la seule énergie d'excitation vibrationnelle ou électronique des fragments $\mathrm{C}_{2} \mathrm{H}_{3}, \mathrm{C}_{2} \mathrm{H}_{2}, \mathrm{C}_{2} \mathrm{H}$ et $\mathrm{H}_{2}$.

A priori, nous ne sommes pas en mesure de décider lequel des trois mécanismes est responsable des seuils 
Published in: Bulletin de la Classe des Sciences. Académie Royale de Belgique (1970), vol. LVI, pp. $788-817$.

Status: Postprint (Author's Version)

observés à 7,5 eV et à 9,5 eV.

FIG. 7.

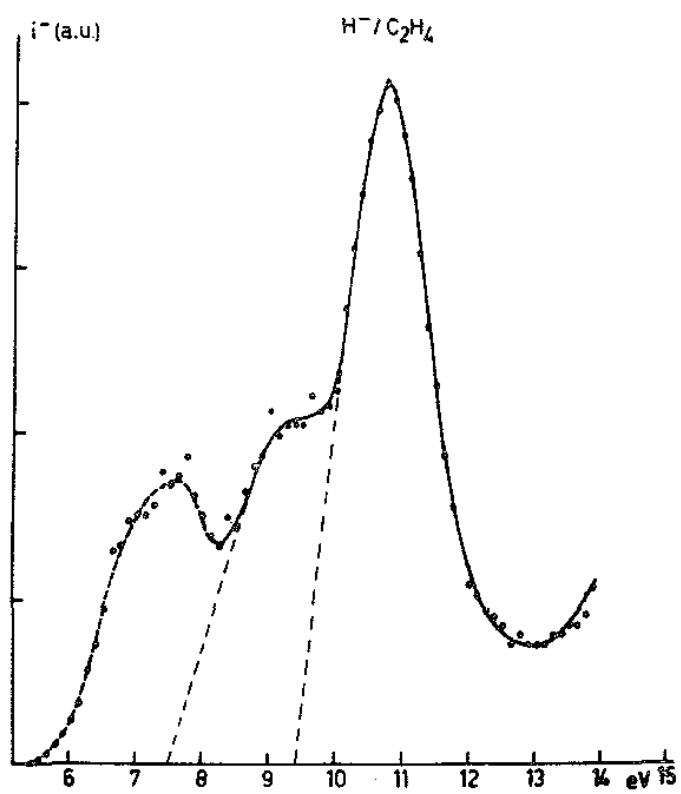

\section{b) L'ion $\boldsymbol{C H}$ (figure 8)}

Le mécanisme le plus probable, dormant naissance à l'ion $\mathrm{CH}^{-}$par attachement électronique dissociatif à $9,0 \mathrm{eV}$, est

$$
\mathrm{C}_{2} \mathrm{H}_{4}+\mathrm{e}^{-} \rightarrow \mathrm{CH}^{-}+\mathrm{CH}_{2}+\mathrm{H}
$$

FIG. 8.

$\mathrm{CH}^{-} / \mathrm{C}_{2} \mathrm{H}_{4}$

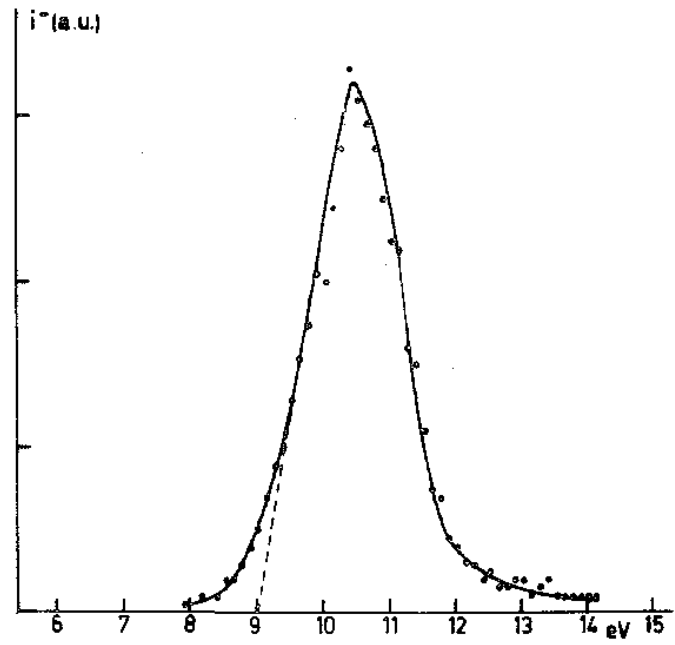


En admettant que l'énergie cinétique emportée par l'ion négatif est nulle, nous calculons une valeur de $\mathrm{EA}(\mathrm{CH})=2,7 \mathrm{eV}$, si nous adoptons la valeur de $\mathrm{D}\left(\mathrm{CH}_{2}=\mathrm{CH}_{2}\right)=(7,6 \pm 0,3) \mathrm{eV}$ [9] et $\mathrm{D}(\mathrm{CH}-\mathrm{H})=4,23 \mathrm{eV}[8]$.

\section{c) L'ion $\boldsymbol{C}_{2} \boldsymbol{H}^{-}$(figure 9)}

Les seuils des deux processus d'attachement électronique dissociatif qui donnent naissance à l'ion $\mathrm{C}_{2} \mathrm{H}^{-}$à partir de $\mathrm{C}_{2} \mathrm{H}_{4}$ sont situés à 6,9 et $9,9 \mathrm{eV}$.

A partir des deux mécanismes qui permettent d'interpréter l'apparition de l'ion $\mathrm{C}_{2} \mathrm{H}^{-}$:

$$
\begin{aligned}
& \mathrm{C}_{2} \mathrm{H}_{4}+\mathrm{e}^{-} \rightarrow \mathrm{C}_{2} \mathrm{H}^{-}+\mathrm{CH}_{2}+\mathrm{H} \\
& \mathrm{C}_{2} \mathrm{H}_{4}+\mathrm{e}^{-} \rightarrow \mathrm{C}_{2} \mathrm{H}^{-}+3 \mathrm{H}
\end{aligned}
$$

nous calculons, sur la base du premier seuil, une chaleur de formation de l'ion $\mathrm{C}_{2} \mathrm{H}^{-}$de $5,2 \mathrm{eV}$ et $3,66 \mathrm{eV}$ respectivement; les valeurs correspondantes de l'électroaffinité de $\mathrm{C}_{2} \mathrm{H}$ sont $\mathrm{EA}_{1}\left(\mathrm{C}_{2} \mathrm{H}\right)=(0 \pm 0,3)$ eV et EA $\mathrm{EA}_{2}\left(\mathrm{C}_{2} \mathrm{H}\right)$ $=(1,6 \pm 0,3) \mathrm{eV}$. A $6,9 \mathrm{eV}$, le mécanisme le plus probable est:

$$
\mathrm{C}_{2} \mathrm{H}_{4}+\mathrm{e}^{-} \rightarrow \mathrm{C}_{2} \mathrm{H}^{-}+\mathrm{H}_{2}+\mathrm{H}
$$

où l'ion $\mathrm{C}_{2} \mathrm{H}^{-}$apparaîtrait dans un état électronique excité en emportant une certaine quantité d'énergie cinétique [13].

FIG. 9.

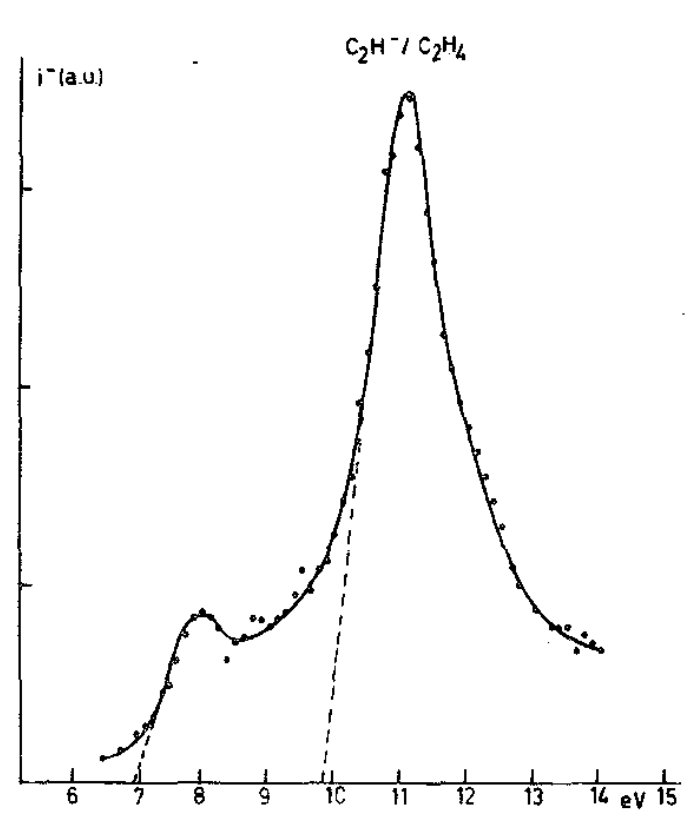

Le processus correspondant au seuil de 9,9 eV serait dû au mécanisme :

$$
\mathrm{C}_{2} \mathrm{H}_{4}+\mathrm{e}^{-} \rightarrow \mathrm{C}_{2} \mathrm{H}^{-}+3 \mathrm{H}
$$

où l'ion $\mathrm{C}_{2} \mathrm{H}^{-}$apparaît dans l'état fondamental avec une faible quantité d'énergie cinétique. 


\section{LES DERIVES HALOGENES DE L'ETHYLENE}

\section{Le chlorure de vinyle $\mathrm{C}_{2} \mathrm{H}_{3} \mathrm{Cl}$}

Parmi les ions composant le spectre de masse des ions négatifs du monochloréthylène, nous avons étudié le processus d'attachement électronique dissociatif pour les ions $\mathrm{H}^{-}$et $\mathrm{C}_{2} \mathrm{H}^{-}$dont nous avons repris les potentiels d'apparition dans le tableau VII. Les autres ions, de faible intensité, ne pouvant faire l'objet d'une étude de leur potentiel d'apparition, ne sont pas mentionnés ici.

TABLEAU VII. Énergie de seuil (en eV) des ions négatifs apparaissant dans $\mathrm{C}_{2} \mathrm{H}_{3} \mathrm{Cl}$.

\begin{tabular}{|c|c|c|c|}
\hline \multicolumn{2}{|c|}{$\mathbf{H}^{-}$} & \multicolumn{2}{c|}{$\mathbf{C}_{2} \mathbf{H}^{-}$} \\
\hline P.A. & $\boldsymbol{\Delta E}$ & P.A. & $\boldsymbol{\Delta E}$ \\
\hline $7,5 \pm 0,2$ & 0,7 & $6,17 \pm 0,05$ & \\
\hline $8,2 \pm 0,1$ & & Maxima : & \\
\hline & & $8,9 \pm 0,1$ & \\
\hline Maximum : & & $10,1 \pm 0,1$ & 1,2 \\
\hline $11,6 \pm 0,1$ & & $10,5 \pm 0,05$ & 0,4 \\
\hline & & $11,4 \pm 0,1$ & 0,9 \\
\hline & & $12,5 \pm 0,4$ & 1,1 \\
\hline
\end{tabular}

\section{DISCUSSION}

a) L'ion $\boldsymbol{H}$ (figure 10)

FIG. 10.

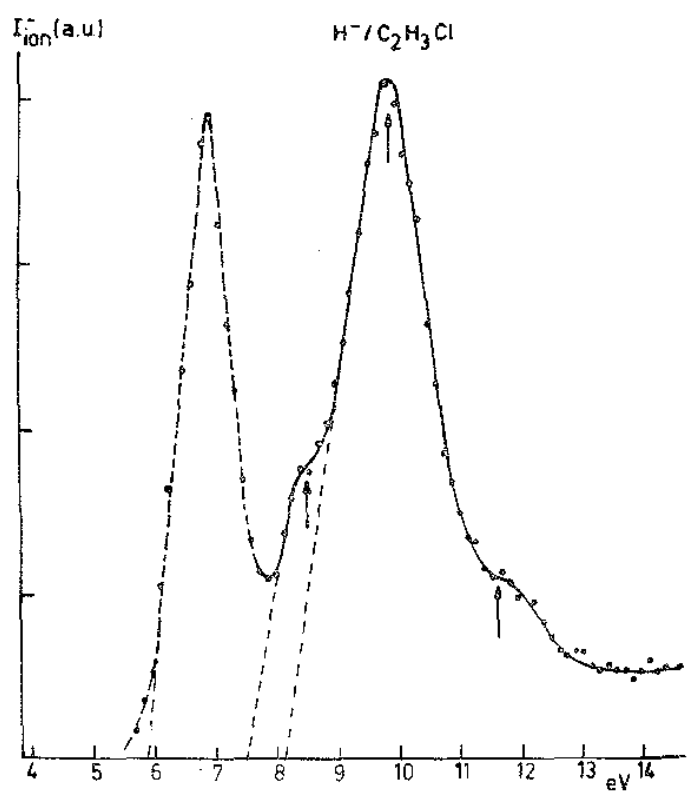

Le pic prenant naissance à $6,1 \mathrm{eV}$ est attribué à l'ion $\mathrm{H}^{-}$provenant de l'eau résiduelle de la source d'ions. L'intensité du pic d'attachement électronique dissociatif de $\mathrm{H}^{-}$provenant de $\mathrm{C}_{2} \mathrm{H}_{3} \mathrm{C} 1$ est de faible intensité. Nous déterminons deux potentiels d'apparition: à $(7,5 \pm 0,2) \mathrm{eV}$ et à $(8,2 \pm 0,1) \mathrm{eV}$.

Parmi les mécanismes dont le seuil d'apparition a pu être calculé a priori, deux peuvent donner naissance à l'ion $\mathrm{H}^{-}$par attachement électronique dissociatif: 


$$
\begin{gathered}
\mathrm{C}_{2} \mathrm{H}_{3} \mathrm{Cl}+\mathrm{e}^{-} \rightarrow \mathrm{H}^{-}+\mathrm{C}_{2} \mathrm{H}_{2}+\mathrm{Cl} \\
\mathrm{C}_{2} \mathrm{H}_{3} \mathrm{Cl}+\mathrm{e}^{-} \rightarrow \mathrm{H}^{-}+\mathrm{C}_{2} \mathrm{H}+\mathrm{HCl}
\end{gathered}
$$

Les potentiels d'apparition calculés sont 5,41 eV et 5,97 eV respectivement.

Deux interprétations permettent de rendre compte des deux potentiels critiques mesurés:

(i) l'apparition de la molécule d'acétylène dans différents états vibrationnels excités, ou l'apparition de l'ion $\mathrm{H}^{-}$aux dépens de l'ion moléculaire négatif dans divers états vibrationnels [mécanisme de «prédissociation » [15] (figure 11)]; l'ion $\mathrm{H}^{-}$est porteur d'énergie cinétique.

(ii) si le mécanisme (2) rend compte des seuils à $7,5 \mathrm{eV}$ et $8,2 \mathrm{eV}$, le radical $\mathrm{C}_{2} \mathrm{H}$ peut apparaitre à des états vibroniques excités. L'épaulement que nous avons caractérisé par un maximum situé à $(11,6 \pm 0,1) \mathrm{eV}$ est vraisemblablement dû au mécanisme:

$$
\mathrm{C}_{2} \mathrm{H}_{3} \mathrm{Cl}+\mathrm{e}^{-} \rightarrow \mathrm{C}_{2} \mathrm{H}+\mathrm{H}+\mathrm{Cl}+\mathrm{H}^{-}
$$

Si nous admettons $\mathrm{D}\left(\mathrm{C}_{2} \mathrm{H}-\mathrm{H}\right)=4,9 \mathrm{eV}[8]$ et $\mathrm{D}(\mathrm{H}-\mathrm{Cl})=4,43 \mathrm{eV}$ [9], nous obtenons à partir des deux mécanismes possibles (1) et (2), les seuils à $10,31 \mathrm{eV}$ et $10,51 \mathrm{eV}$ pour le processus (3). L'ion $\mathrm{H}^{-}$sera porteur d'une certaine quantité d'énergie cinétique.

FIG. 11.

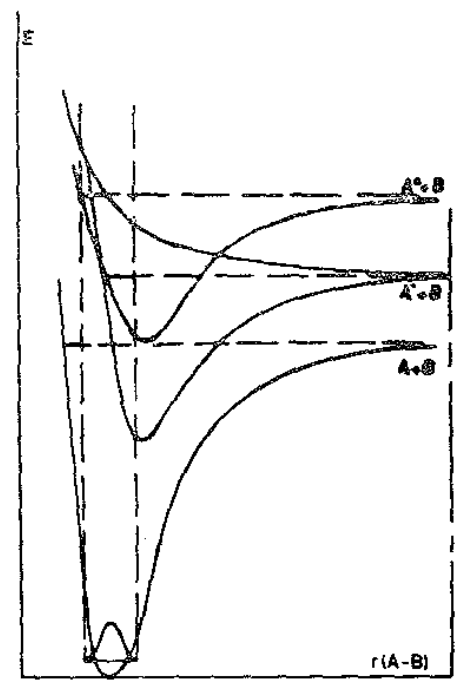

\section{b) L'ion $\boldsymbol{C}_{2} \boldsymbol{H}$ (figure 12)}

Selon celle des deux réactions pouvant interpréter le seuil observé à $(6,17 \pm 0,05) \mathrm{eV}$,

$$
\begin{gathered}
\mathrm{C}_{2} \mathrm{H}_{3} \mathrm{Cl}+\mathrm{e}^{-} \rightarrow \mathrm{C}_{2} \mathrm{H}^{-}+\mathrm{H}+\mathrm{HCl} \\
\mathrm{C}_{2} \mathrm{H}_{3} \mathrm{Cl}+\mathrm{e}^{-} \rightarrow \mathrm{C}_{2} \mathrm{H}^{-}+\mathrm{H}_{2}+\mathrm{Cl}
\end{gathered}
$$

nous obtenons pour la chaleur de formation de $\mathrm{C}_{2} \mathrm{H}: \Delta \mathrm{U}_{\mathrm{f}}\left(\mathrm{C}_{2} \mathrm{H}\right)=4,56 \mathrm{eV}$ ou $\Delta \mathrm{U}_{\mathrm{f}}\left(\mathrm{C}_{2} \mathrm{H}\right)=4,62 \mathrm{eV}$ respectivement. L'électroaffinité déduite de ces grandeurs est $\mathrm{EA}\left(\mathrm{C}_{2} \mathrm{H}\right)=0,6 \mathrm{eV}$.

L'étude de l'apparition de l'ion $\mathrm{C}_{2} \mathrm{H}^{-}$à partir des molécules $\mathrm{C}_{2} \mathrm{H}_{2}, \mathrm{C}_{2} \mathrm{H}_{4}, \mathrm{C}_{2} \mathrm{H}_{3} \mathrm{Cl}$ et $\mathrm{C}_{2} \mathrm{H}_{3} \mathrm{~F}$ par les 
processus d'attachement électronique et de paire d'ions [13] nous conduit à la valeur $\mathrm{EA}\left(\mathrm{C}_{2} \mathrm{H}\right)=(2,1 \pm 0,3) \mathrm{eV}$. Il est vraisemblable que l'ion $\mathrm{C}_{2} \mathrm{H}^{-}$apparaît par l'un des deux mécanismes ci-dessus, emportant une certaine quantité d'énergie cinétique et/ou dans un état électronique excité.

FIG. 12.

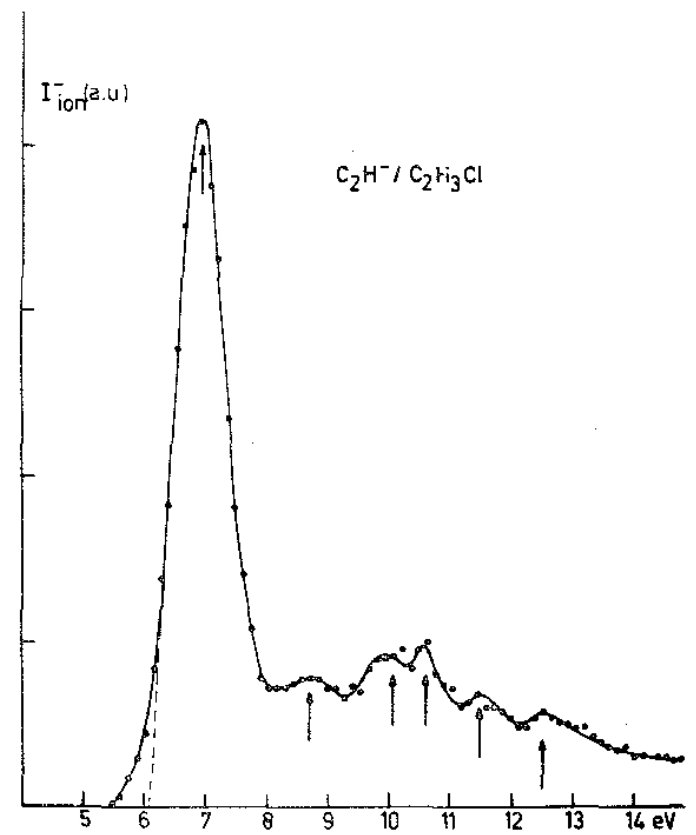

Les processus situés au-delà du premier seuil sont de faible intensité. L'ion négatif peut apparaître dans plusieurs états excités. Les fragments $\mathrm{H}_{2}$ et $\mathrm{HC} 1$ apparaissent à l'état fondamental ou à divers états vibrationnels excités. Nous ne pouvons pas exclure que le processus dont le maximum est situé à 11,4 eV soit dû au mécanisme:

$$
\mathrm{C}_{2} \mathrm{H}_{3} \mathrm{Cl}+\mathrm{e}^{-} \rightarrow \mathrm{C}_{2} \mathrm{H}^{-}+2 \mathrm{H}+\mathrm{Cl}
$$

dont le seuil se situerait à $(9,1+0,3) \mathrm{eV}$, si nous adoptons la valeur $\mathrm{EA}\left(\mathrm{C}_{2} \mathrm{H}\right)=(2,1 \pm 0,3) \mathrm{eV}[13]$.

\section{Le fluorure de vinyle $\mathrm{C}_{2} \mathrm{H}_{3} \mathrm{~F}$}

Dans le tableau VIII, nous avons repris les potentiels d'apparition des principaux ions négatifs composant le spectre de niasse du monofluoroéthylène. Certains processus sont caractérisés par la position du maximum lorsque la résolution ne permet pas de pratiquer la méthode d'extrapolation linéaire.

TABLEAU VIII. Énergie de seuil (eV) des ions négatifs apparaissant dans $\mathrm{C}_{2} \mathrm{H}_{3} \mathrm{~F}$.

\begin{tabular}{|c|c|c|c|c|c|c|}
\hline \multicolumn{2}{|l|}{ H- } & \multicolumn{2}{|l|}{$\mathbf{F}^{-}$} & \multicolumn{2}{|l|}{$\mathrm{C}_{2}^{-}$} & $\mathrm{C}_{2} \mathrm{H}^{-}$ \\
\hline P.A. & $\Delta E$ & P.A. & $\Delta E$ & P.A. & $\Delta \mathrm{E}$ & P.A. \\
\hline $8,6 \pm 0,1$ & \multirow{8}{*}{$\begin{array}{l}0,6 \\
0,7 \\
0,7\end{array}$} & $1,5 \pm 0,1$ & \multirow{8}{*}{$\begin{array}{l}0,7 \\
0,2 \\
0,6\end{array}$} & $6,8 \pm 0,1$ & \multirow{8}{*}{$\begin{array}{c}0,7 \\
0,8 \\
0,8\end{array}$} & $6,9 \pm 0,1$ \\
\hline Maxima & & Maxima: & & $9,1 \pm 0,08$ & & $9,1 \pm 0,1$ \\
\hline $11,1 \pm 0,2$ & & $7,5 \pm 0,05$ & & Maxima: & & Maximum \\
\hline $11,7 \pm 0,1$ & & $7,9 \pm 0,1$ & & $11,4 \pm 0,05$ & & $11,2 \pm 0,2$ \\
\hline $12,4 \pm 0,2$ & & $9,5 \pm 0,2$ & & $12,1 \pm 0,05$ & & \\
\hline \multirow[t]{3}{*}{$13,1 \pm 0,2$} & & $10,2 \pm 0,06$ & & $12,9 \pm 0,2$ & & \\
\hline & & $10,4 \pm 0,1$ & & $13,7 \pm 0,2$ & & \\
\hline & & $11,0 \pm 0,2$ & & & & \\
\hline
\end{tabular}




\section{DISCUSSION}

a) L'ion $\boldsymbol{H}$ (figure 13)

FIG. 13.

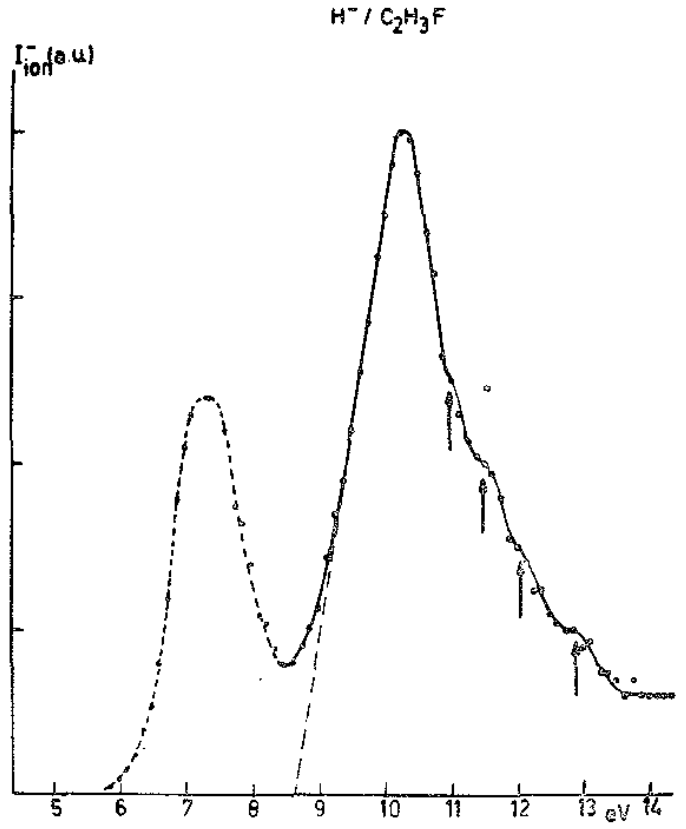

La courbe d'efficacité d'attachement électronique dissociatif donnant lieu à l'apparition de $\mathrm{H} \sim$ se présente sous la forme d'un large pic comportant un maximum bien défini suivi de quatre épaulements à espacements réguliers d'environ $0,7 \mathrm{eV}$.

Deux processus, parmi ceux pour lesquels $\mathrm{PA}_{\text {calc }} \leq \mathrm{PA}_{\text {exp }}$ et dont le calcul a priori est possible, retiendront notre attention pour interpréter le seuil à $(8,6 \pm 0,1) \mathrm{eV}$ :

$$
\begin{aligned}
& \mathrm{C}_{2} \mathrm{H}_{3} \mathrm{~F}+\mathrm{e}^{-} \rightarrow \mathrm{C}_{2} \mathrm{H}_{2}+\mathrm{F}+\mathrm{H}^{-} \\
& \mathrm{C}_{2} \mathrm{H}_{3} \mathrm{~F}+\mathrm{e}^{-} \rightarrow \mathrm{C}_{2} \mathrm{H}+\mathrm{HF}+\mathrm{H}^{-}
\end{aligned}
$$

Le potentiel d'apparition calculé à partir du mécanisme (1) est situé $5,89 \mathrm{eV}$; le seuil du deuxième processus serait situé à $5,02 \mathrm{eV}$.

Les différences entre $\mathrm{PA}_{\text {calc }}$ et le seuil expérimenta! sont $2,7 \mathrm{eV}$ et 3,6 eV respectivement. 11 est vraisemblable que cet excès d'énergie correspond à l'excitation de la molécule $\mathrm{C}_{2} \mathrm{H}_{2}$ ou $\mathrm{HF}$ ou du radical $\mathrm{C}_{2} \mathrm{H}$ et/ou à l'énergie cinétique emportée par l'ion $\mathrm{H}^{-}$. Si le processus (1) est le plus simple, il ne nous est cependant pas possible de décider lequel des deux mécanismes est en cause à $8,6 \mathrm{eV}$.

La structure fine observée à la suite du maximum correspondant au mécanisme principal peut être interprétée par une décomposition de l'ion moléculaire par une «prédissociation» (figure 11). Un tel phénomène fut observé antérieurement dans l'hydrogène par Dowell et Sharp [15].

\section{b) $L$ 'ion $F$ (figure 14)}

La courbe d'efficacité d'attachement électronique dissociatif montre l'existence de trois processus. Un premier pic, le plus intense, correspond à un seuil situé à $(1,5 \pm 0,1) \mathrm{eV}$. A priori, un seul mécanisme est possible:

$$
\mathrm{C}_{2} \mathrm{H}_{3} \mathrm{~F}+\mathrm{e}^{-} \rightarrow \mathrm{C}_{2} \mathrm{H}_{3}+\mathrm{F}^{-}
$$


dont le seuil d'apparition calculé est situé à 1,31 eV. L'ion F־emporterait une faible quantité d'énergie cinétique.

Pour permettre une évaluation approximative du seuil des deux autres processus, nous avons extrapolé la plus grande pente du flanc de basse énergie de chacun des pics correspondants. Le deuxième processus a un seuil probable situé à $6,1 \mathrm{eV}$ qui pourrait correspondre à:

$$
\begin{aligned}
& \mathrm{C}_{2} \mathrm{H}_{3} \mathrm{~F}+\mathrm{e}^{-} \rightarrow \mathrm{C}_{2} \mathrm{H}_{2}+\mathrm{H}+\mathrm{F}^{-}\left(\mathrm{PA}_{\text {calc }}=3,19 \mathrm{eV}\right) \\
& \mathrm{C}_{2} \mathrm{H}_{3} \mathrm{~F}+\mathrm{e}^{-} \rightarrow \mathrm{C}_{2} \mathrm{H}+\mathrm{H}_{2}+\mathrm{F}^{-}\left(\mathrm{PA}_{\text {calc }}=3,69 \mathrm{eV}\right)
\end{aligned}
$$

Dans les deux cas, l'ion $\mathrm{F}^{-}$apparaîtrait avec énergie cinétique et/ou le radical $\mathrm{C}_{2} \mathrm{H}$ ou les molécules $\mathrm{C}_{2} \mathrm{H}_{2}$ et $\mathrm{H}_{2}$ à un état vibrationnel excité.

FIG. 14.

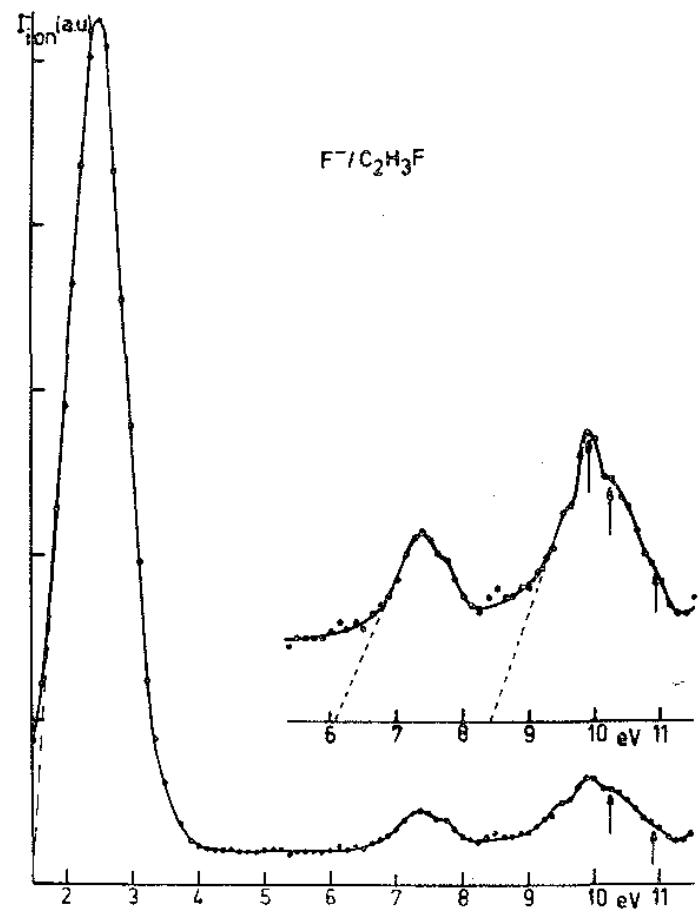
mécanisme:

Le seuil probable du troisième processus est situé à $8,5 \mathrm{eV}$ : le potentiel d'apparition calculé à partir du

$$
\mathrm{C}_{2} \mathrm{~F}_{3} \mathrm{H}+\mathrm{e}^{-} \rightarrow \mathrm{C}_{2} \mathrm{H}+2 \mathrm{H}+\mathrm{F}^{-}
$$

est situé à $8,2 \mathrm{eV}$. Il est vraisemblable que les trois maxima observés aux intervalles $0,7 \mathrm{eV}, 0,2 \mathrm{eV}$ et $0,6 \mathrm{eV}$ soient dus à l'apparition de l'ion $\mathrm{F}^{-}$à partir de niveaux vibrationnels de l'ion moléculaire par le mécanisme de " prédissociation $»[15]$.

\section{c) L'ion $\mathrm{C}_{2}^{-}$(figure 15)}

L'allure générale de la courbe d'ionisation est semblable à celle que nous avons observée pour l'ion $\mathrm{F}^{-}$: un processus simple précédant un processus complexe.

Le premier seuil d'apparition est situé à $(6,8 \pm 0,1) \mathrm{eV}$. Lorsque nous calculons la chaleur de formation de l'ion $\mathrm{C}_{2}^{-}$à partir du mécanisme

$$
\mathrm{C}_{2} \mathrm{H}_{3} \mathrm{~F}+\mathrm{e}^{-} \rightarrow \mathrm{C}_{2}^{-}+\mathrm{H}_{2}+\mathrm{HF}
$$


nous obtenons $\Delta \mathrm{U}_{\mathrm{f}}\left(\mathrm{C}_{2}^{-}\right)=8,43 \mathrm{eV}$, La valeur de l'électroaffinité que nous en déduisons est $\mathrm{EA}\left(\mathrm{C}_{2}\right)=(0,2 \pm 0,1) \mathrm{eV}$. Cette valeur de EA peut correspondre à l'apparition de l'ion $\mathrm{C}_{2}{ }^{-}$à un état électronique excité. Dans la molécule $\mathrm{N}_{2}{ }^{+}$, isoélectonique de $\mathrm{C}_{2}^{-}$, l'état $\mathrm{B}^{2} \Sigma^{+}$est situé à 3,2 eV au-dessus de l'état fondamental [9].

Le seul mécanisme permettant d'interpréter le deuxième seuil à $(9,1 \pm 0,1) \mathrm{eV}$ est

$$
\mathrm{C}_{2} \mathrm{H}_{3} \mathrm{~F}+\mathrm{e}^{-} \rightarrow \mathrm{C}_{2}^{-}+2 \mathrm{H}+\mathrm{HF}
$$

La valeur de l'électroaffinité du radical $\mathrm{C}_{2}$ obtenue est $\mathrm{EA}\left(\mathrm{C}_{2}\right)=(2,4 \pm 0,1)$ eV correspondant à l'apparition de l'ion $\mathrm{C}_{2}^{-}$dans un état électronique excité et/ou est porteur d'énergie cinétique. Dans la molécule $\mathrm{N}_{2}{ }^{+}$, isoélectronique de $\mathrm{C}_{2}^{-}$, l'état $\mathrm{A}^{2} \Pi$ est situé à $1,1 \mathrm{eV}$ au-dessous de l'état $\mathrm{X}^{2} \Sigma_{\mathrm{g}}^{+}$[91. Il n'est pas exclu que l'ion $\mathrm{C}_{2}{ }^{-}$ soit porteur d'énergie cinétique (l'énergie minimum d'excitation électronique des atomes $\mathrm{H}$ et de la molécule HF est de $9 \mathrm{eV})$.

\section{FIG. 15.}

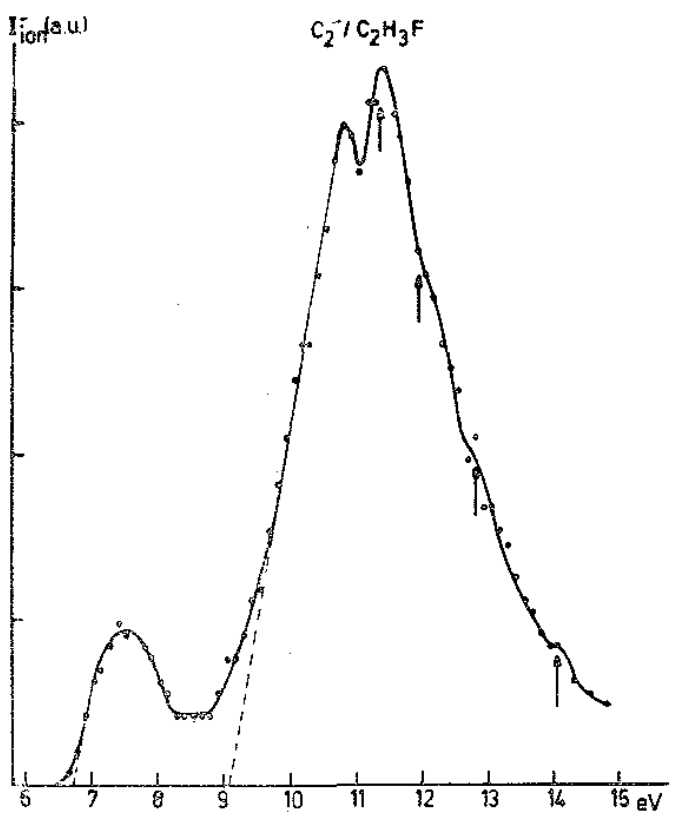

Les maxima situés à $11,4 \mathrm{eV}, 12,1 \mathrm{eV}, 12,9 \mathrm{eV}$ et $13,7 \mathrm{eV}$ peuvent être interprétés par l'apparition de $\mathrm{C}_{2}{ }^{-}$ à partir de l'ion moléculaire par le mécanisme de «prédissociation ». L'espacement des épaulements, d'environ 0,7 $\mathrm{eV}$ est le même que celui relevé dans la courbe d'attachement électronique dissociatif de $\mathrm{H}^{-}$dans $\mathrm{C}_{2} \mathrm{H}_{3} \mathrm{~F}$ et dans un même domaine d'énergie (entre $11 \mathrm{eV}$ et $13 \mathrm{eV}$ ). Il n'est pas exclu que la même surface d'énergie potentielle relative à l'ion moléculaire $\mathrm{C}_{2} \mathrm{H}_{3} \mathrm{~F}^{-}$soit recoupée par deux surfaces répulsives dont l'une donne naissance à l'ion $\mathrm{C}_{2}^{-}$.

\section{d) L'ion $\mathrm{C}_{2} \mathrm{H}$ (figure 16)}

Le seul mécanisme qui permette d'interpréter le premier seuil

$$
\mathrm{C}_{2} \mathrm{H}_{3} \mathrm{~F}+\mathrm{e}^{-} \rightarrow \mathrm{C}_{2} \mathrm{H}^{-}+\mathrm{H}_{2}+\mathrm{F}
$$

fournit une chaleur de formation $\Delta \mathrm{U}_{\mathrm{f}}\left(\mathrm{C}_{2} \mathrm{H}^{-}\right)=(4,9 \pm 0,1)$ eV et nous en déduisons $\mathrm{EA}\left(\mathrm{C}_{2} \mathrm{H}\right)=0,2 \mathrm{eV}$. Cette valeur de EA peut correspondre à l'apparition de l'ion $\mathrm{C}_{2} \mathrm{H}^{-}$dans un état électronique excité [13], porteur d'énergie cinétique.

A 9,1 eV l'ion $\mathrm{C}_{2} \mathrm{H}^{-}$peut apparaître par le mécanisme:

$$
\mathrm{C}_{2} \mathrm{H}_{3} \mathrm{~F}+\mathrm{e}^{-} \rightarrow \mathrm{C}_{2} \mathrm{H}^{-}+2 \mathrm{H}+\mathrm{F}
$$


La valeur de $\mathrm{EA}\left(\mathrm{C}_{2} \mathrm{H}\right)$ obtenue à partir de ce mécanisme est $(2,5 \pm 0,1) \mathrm{eV}$, proche de la valeur $(2,1 \pm 0,2) \mathrm{eV}$ déterminée à partir de $\mathrm{C}_{2} \mathrm{H}_{2}$. L'épaulement observé à $11,2 \mathrm{eV}$ peut être dû à l'apparition de

$$
\mathrm{C}_{2} \mathrm{H}_{3} \mathrm{~F}+\mathrm{e}^{-} \rightarrow \mathrm{C}_{2} \mathrm{H}^{-*}+2 \mathrm{H}+\mathrm{F} \text {. }
$$

FIG. 16.

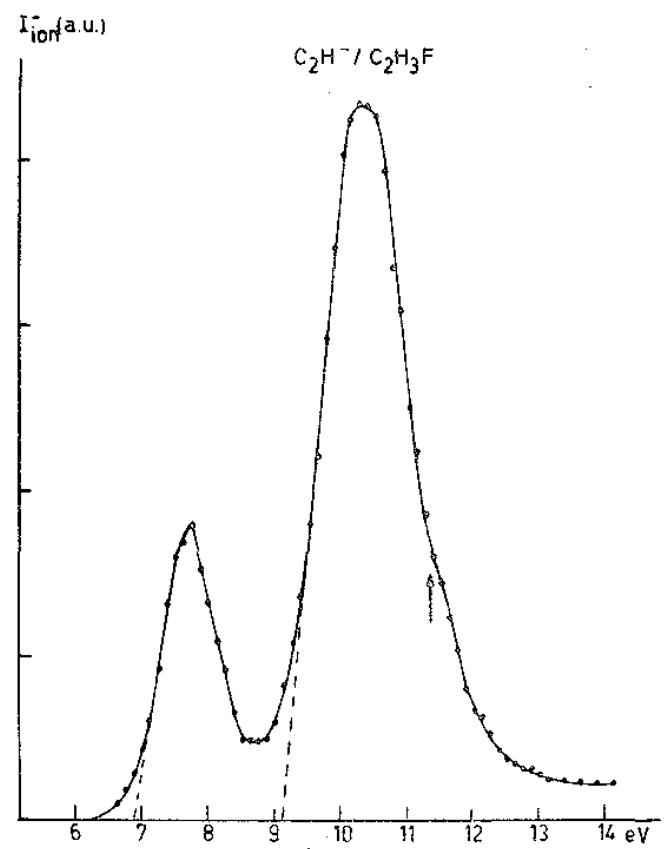

\section{CONCLUSIONS}

\begin{tabular}{|c|c|c|}
\hline & P.A.(eV) & Mécanismes proposés \\
\hline \multirow[t]{4}{*}{ A. } & $8,2 \pm 0,2$ & \multirow[t]{2}{*}{$\mathrm{CH}_{4}+\mathrm{e}^{-} \rightarrow \mathrm{H}^{-}+\mathrm{H}_{2}+\mathrm{CH}$} \\
\hline & $9,4 \pm 0,15$ & \\
\hline & $9,7 \pm 0,2$ & $\mathrm{CH}_{4} \pm \mathrm{e}^{-} \rightarrow \mathrm{CH}^{-}+\mathrm{H}_{2}+\mathrm{H}$ \\
\hline & $9,3 \pm 0,3$ & $\mathrm{CH}_{4}+\mathrm{e}^{-} \rightarrow \mathrm{CH}_{2}+2 \mathrm{H}$ \\
\hline \multirow[t]{5}{*}{ B. } & $7,2 \pm 0,1$ & $\mathrm{C}_{2} \mathrm{H}_{2}+\mathrm{e}^{-} \rightarrow \mathrm{H}^{-}+\mathrm{C}_{2} \mathrm{H}^{*}$ \\
\hline & $7,6 \pm 0,2$ & $\mathrm{C}_{2} \mathrm{H}_{2}+\mathrm{e}^{-} \rightarrow \mathrm{C}_{2}^{-}+2 \mathrm{H}^{*}$ \\
\hline & $11,6 \pm 0,2$ & $\rightarrow \mathrm{C}_{2}^{-*}+2 \mathrm{H}$ \\
\hline & $12,2 \pm 0,2$ & \\
\hline & $2,8 \pm 0,2$ & $\mathrm{C}_{2} \mathrm{H}_{2}+\mathrm{e}^{-} \rightarrow \mathrm{C}_{2} \mathrm{H}^{-}+\mathrm{H}$ \\
\hline \multirow[t]{5}{*}{ C. } & $7.5-4-0,3$ & $\mathrm{C}_{4} \mathrm{H}_{2}+\mathrm{e}^{-} \rightarrow \mathrm{H}^{-}+(?)$ \\
\hline & $9.6 \pm 0,1$ & \\
\hline & $9,0 \pm 0,1$ & $\mathrm{C}_{2} \mathrm{H}_{4}+\mathrm{e}^{-} \rightarrow \mathrm{CH}^{-}+\mathrm{CH}_{2}+\mathrm{H}$ \\
\hline & $6,9 \pm 0,1$ & $\mathrm{C}_{2} \mathrm{H}_{4}+\mathrm{e}^{-} \rightarrow \mathrm{C}_{2} \mathrm{H}^{-}+\mathrm{H}_{2}+\mathrm{H}$ \\
\hline & $9,9 \pm 0,05$ & $\rightarrow \mathrm{C}_{2} \mathrm{H}^{-}+3 \mathrm{H}$ \\
\hline \multirow[t]{4}{*}{ D. } & $7,5 \pm 9,2$ & $\mathrm{C}_{2} \mathrm{H}_{3} \mathrm{Cl} 1+\mathrm{e}^{-} \rightarrow \mathrm{H}^{-}+(?)$ \\
\hline & $8,2 \pm 0,1$ & \\
\hline & $6,17 \pm 0,05$ & $\mathrm{C}_{2} \mathrm{H}_{3} \mathrm{Cl}+\mathrm{e}^{-} \rightarrow \mathrm{C}_{2} \mathrm{H}^{-}+\mathrm{H}$ \\
\hline & & $\left(\mathrm{ou} \mathrm{H}_{2}\right)+\mathrm{HCl}(\mathrm{Cl})$ \\
\hline \multirow[t]{6}{*}{ E. } & $8,6 \pm 0,1$ & $\mathrm{C}_{2} \mathrm{H}_{3} \mathrm{~F}+\mathrm{e}^{-} \rightarrow \mathrm{H}^{-}+?$ \\
\hline & $1,5 \pm 0,1$ & $\mathrm{C}_{2} \mathrm{H}_{3} \mathrm{~F}+\mathrm{e}^{-} \rightarrow \mathrm{C}_{2} \mathrm{H}_{3}+\mathrm{F}^{-}$ \\
\hline & $6,8 \pm 0,1$ & $\mathrm{C}_{2} \mathrm{H}_{3} \mathrm{~F}+\mathrm{e}^{-} \rightarrow \mathrm{C}_{2}^{-*}+\mathrm{H}_{2}+\mathrm{HF}$ \\
\hline & $9,1 \pm 0,08$ & $\rightarrow \mathrm{C}_{2}^{-* *}+2 \mathrm{H}+\mathrm{HF}$ \\
\hline & $6,9 \pm 0,1$ & $\mathrm{C}_{2} \mathrm{H}_{3} \mathrm{~F}+\mathrm{e}^{-} \rightarrow \mathrm{C}_{2} \mathrm{H}^{*}+\mathrm{H}_{2}+\mathrm{F}$ \\
\hline & $9,1 \pm 0,1$ & $\rightarrow \mathrm{C}_{2} \mathrm{H}^{-}+2 \mathrm{H}+\mathrm{F}$ \\
\hline
\end{tabular}


Nous avons rassemblé dans le tableau suivant les conclusions relatives à l'étude de l'apparition d'ions négatifs par le processus d'attachement électronique dissociatif dans $\mathrm{CH}_{4}, \mathrm{C}_{2} \mathrm{H}_{2}$ et $\mathrm{C}_{2} \mathrm{H}_{3} \mathrm{X}(\mathrm{X}=\mathrm{H}, \mathrm{Cl}$ et $\mathrm{F})$.

L'examen auquel nous venons de nous livrer au sujet de l'apparition des ions négatifs par le processus d'attachement électronique dissociatif dans des molécules polyatomiques a montré que l'interprétation des seuils observés est plus malaisée que dans les molécules biatomiques. L'origine de ces difficultés est double:

$1^{\circ}$ Le nombre élevé de mécanismes pouvant interpréter un même seuil d'apparition;

$2^{\circ}$ Les lacunes dans la connaissance de l'électroaffinité, de la chaleur de formation et du spectre électronique de nombreux radicaux. Toutefois, nos mesures nous ont permis de tenter une évaluation de l'electroaffinité des radicaux $\mathrm{C}_{2}$ et $\mathrm{C}_{2} \mathrm{H}$ et de mettre en évidence l'existence probable d'états électroniques excités pour les ions négatifs correspondants $\mathrm{C}_{2}^{-}$et $\mathrm{C}_{2} \mathrm{H}^{-}$.

\section{a) L'électroaffinité de $C_{2}$}

Dans le tableau IX, nous avons résumé les conclusions qui résultent de l'étude que nous avons consacrée à l'apparition de l'ion $\mathrm{C}_{2}$.

\section{TABLEAU IX.}

\begin{tabular}{|c|c|c|c|}
\hline Mécanismes & P.A.(eV) & EA(eV) & \\
\hline $\mathrm{C}_{2} \mathrm{H}_{2}+\mathrm{e}^{-} \rightarrow \mathrm{C}_{2}^{-}+2 \mathrm{H}$ & $7,5 \pm 0,2$ & $3,3 \pm 0,2$ & $(\alpha)$ \\
\hline $\mathrm{C}_{2} \mathrm{H}_{3} \mathrm{~F}+\mathrm{e}^{-} \rightarrow \mathrm{C}_{2}^{-}+\mathrm{H}_{2}+\mathrm{HF}$ & $6,8 \pm 0,1$ & $0,2 \pm 0,1$ & $(\beta)$ \\
\hline $\mathrm{C}_{2}^{-}+2 \mathrm{H}+\mathrm{HF}$ & $9,1 \pm 0,1$ & $2,4 \pm 0,1$ & $(\gamma)$ \\
\hline
\end{tabular}

Par sublimation du graphite, Honig [14] détermine deux valeurs de l'électroaffinité, soient 4,0 eV et 3,1 $\mathrm{eV}$; cette dernière valeur est en bon accord avec la valeur obtenue à partir du mécanisme $(\mathrm{a})$, soit $(3,3 \pm 0,3) \mathrm{eV}$.

A partir des données relatives à la molécule $\mathrm{C}_{2} \mathrm{H}_{3} \mathrm{~F}$ nous obtenons deux valeurs $\mathrm{EA}\left(\mathrm{C}_{2}\right)=2,4 \mathrm{eV}$ et 0,2 $\mathrm{eV}$. Pour ces dernières valeurs nous avons été amenés à émettre l'hypothèse de l'existence d'états électroniques excités à $0,9 \mathrm{eV}$ et $3,1 \mathrm{eV}$ au-dessus de l'état fondamental, Dans la molécule $\mathrm{N}_{2}{ }^{+}$, isoélectronique de $\mathrm{C}_{2}{ }^{-}, \mathrm{l}^{\prime}$ état $\mathrm{A}^{2} \Pi$ etB $^{2} \Sigma^{+}$sont situés respectivement à $1,1 \mathrm{eV}$ et $3,3 \mathrm{eV}$ au-dessus de l'état fondamental $\mathrm{X}^{2} \Sigma_{\mathrm{g}}{ }^{+}$.

A partir de la valeur $\mathrm{EA}\left(\mathrm{C}_{2}\right)=(3,3 \pm 0,3) \mathrm{eV}$, il nous est possible d'évaluer l'énergie de dissociation de l'ion $\mathrm{C}_{2}^{-}$par:

$$
\mathrm{D}_{0}^{\circ}\left(\mathrm{C}_{2}^{-}\right)=\mathrm{D}_{0}^{\circ}\left(\mathrm{C}_{2}\right)+\mathrm{EA}\left(\mathrm{C}_{2}\right)-\mathrm{EA}(\mathrm{C})
$$

Si nous admettons que le mécanisme de dissociation soit:

$$
\mathrm{C}_{2}^{-}\left(\mathrm{X}^{2} \Sigma_{g}^{+}\right) \rightarrow \mathrm{C}\left({ }^{3} \mathrm{P}\right)+\mathrm{C}^{-}\left({ }^{4} \mathrm{~S}\right)
$$

nous calculons une énergie de dissociation $\mathrm{D}_{0}{ }^{\circ}\left(\mathrm{C}_{2}{ }^{-}\right)=(8,2 \pm 0,3) \mathrm{eV}$ sachant que $\mathrm{EA}(\mathrm{C})=1,25 \mathrm{eV}[22]$ et $\mathrm{D}_{0}{ }^{\circ}\left(\mathrm{C}_{2}\right)$ $=6,12 \mathrm{eV}[16,17]$. Cette valeur est à rapprocher $\mathrm{de}_{\mathrm{D}_{0}}{ }^{\circ}\left(\mathrm{N}_{2}{ }^{+}\right)=8,72 \mathrm{eV}[9]$, où $\mathrm{N}_{2}{ }^{+}$est isoélectronique de $\mathrm{C}_{2}{ }^{-}$.

\section{b) L'électroaffinité de $C_{2} H$}

\section{TABLEAU X.}

\begin{tabular}{|c|c|c|}
\hline Mécanismes & P.A.(eV) & EA(eV) \\
\hline $\mathrm{C}_{2} \mathrm{H}_{2}+\mathrm{e}^{-} \rightarrow \mathrm{C}_{2} \mathrm{H}^{-}+\mathrm{H}$ & $2,8 \pm 0,1$ & $2,1 \pm 0,1$ \\
\hline $\mathrm{C}_{2} \mathrm{H}_{4}+\mathrm{e}^{-} \rightarrow \mathrm{C}_{2} \mathrm{H}^{-}+\mathrm{H}_{2}+\mathrm{H}$ & $6,9 \pm 0,1$ & $0,0 \pm 0,3$ \\
\hline $\mathrm{C}_{2} \mathrm{H}_{3} \mathrm{C} 1+\mathrm{e}^{-} \rightarrow \mathrm{C}_{2} \mathrm{H}^{-}+\mathrm{H}+\mathrm{HC} 1$ & $6,17 \pm 0,05$ & $0,6 \pm 0,1$ \\
\hline $\mathrm{C}_{2} \mathrm{H}^{-}+\mathrm{H}_{2}+\mathrm{Cl}$ & & \\
\hline $\mathrm{C}_{2} \mathrm{H}_{3} \mathrm{~F}+\mathrm{e}^{-} \rightarrow \mathrm{C}_{2} \mathrm{H}^{-}+\mathrm{H}_{2}+\mathrm{F}$ & $6,9 \pm 0,1$ & $0,2 \pm 0,1$ \\
\hline $\mathrm{C}_{2} \mathrm{H}^{-}+2 \mathrm{H}+\mathrm{F}$ & $9,1 \pm 0,1$ & $2,5 \pm 0,1$ \\
\hline
\end{tabular}


L'apparition de l'ion $\mathrm{C}_{2} \mathrm{H}^{-}$par le processus d'attachement électronique dissociatif a été étudié dans $\mathrm{C}_{2} \mathrm{H}_{2}$, $\mathrm{C}_{2} \mathrm{H}_{4}, \mathrm{C}_{2} \mathrm{H}_{3} \mathrm{~F}$ et $\mathrm{C}_{2} \mathrm{H}_{3} \mathrm{C} 1$. Dans le tableau X nous avons rassemblé les conclusions obtenues.

La valeur la plus probable que nous puissions dégager de ce tableau est $\mathrm{EA}\left(\mathrm{C}_{2} \mathrm{H}\right)=(2,3 \pm 0,2) \mathrm{eV}$. Les deux faibles valeurs de l'électroaffinité de $\mathrm{C}_{2} \mathrm{H}$ ne peuvent être interprétées sans recourir à l'hypothèse de la formation de l'ion $\mathrm{C}_{2} \mathrm{H}^{-}$dans un état vibronique excité.

\section{REMERCIEMENTS}

Que Monsieur le professeur L. D'Or veuille trouver ici l'expression de notre gratitude. Nous tenons à remercier Monsieur J. Momigny pour de nombreuses discussions.

L'acquisition de l'instrument utilisé fut rendue possible grâce à l'intervention financière du Fonds National de la Recherche Fondamentale Collective. Qu'il veuille trouver ici l'expression de notre gratitude.

\section{APPENDICE}

Dans le calcul a priori des potentiels d'apparition des processus d'attachement électronique dissociatif, nous avons adopté pour les grandeurs $\Delta \mathrm{U}_{\mathrm{f}}$ (chaleur de formation), EA (électroaffinité) et $\mathrm{D}$ (énergie de dissociation), les valeurs reprises dans le tableau qui suit:

\begin{tabular}{|c|c|c|c|}
\hline & $\Delta \mathbf{U}_{f}(\mathbf{e V})$ & $\mathbf{E A}(\mathbf{e V})$ & Références \\
\hline $\mathrm{C}_{2} \mathrm{H}_{3} \mathrm{~F}$ & $-1,21$ & & $(18)$ \\
\hline $\mathrm{H}$ & 2,26 & 0,75 & $(20),(10)$ \\
\hline $\mathrm{F}$ & 0,82 & 3,45 & $(23),(19)$ \\
\hline $\mathrm{C}_{2} \mathrm{H}_{2}$ & 2,35 & - & $(19)$ \\
\hline $\mathrm{C}_{2} \mathrm{H}$ & 5,11 & - & $(11)$ \\
\hline $\mathrm{C}_{2}$ & $8,63 \pm 0,1$ & - & $(19)$ \\
\hline $\mathrm{HF}$ & $-2,81$ & - & $(19)$ \\
\hline $\mathrm{C}$ & 7,41 & 1,25 & $(19),(22)$ \\
\hline $\mathrm{CH}_{2}$ & 3,98 & - & $(20)$ \\
\hline $\mathrm{CH}_{\mathrm{CHF}}$ & 6,16 & - & $(19)$ \\
\hline $\mathrm{C}_{2} \mathrm{~F}$ & $0,35(?)$ & - & $(11)$ \\
\hline $\mathrm{C}_{2} \mathrm{H}_{2} \mathrm{~F}$ & $2,86 \pm 0,4$ & - & $(11)$ \\
\hline $\mathrm{C}_{2} \mathrm{H}_{3} \mathrm{C} 1$ & 0,41 & - & $(18)$ \\
\hline $\mathrm{Cl}$ & $-0,31$ & - & $(18)$ \\
\hline $\mathrm{HCl}$ & 1,25 & - & $(19)$ \\
\hline $\mathrm{CF}$ & $-0,95$ & - & $(19)$ \\
\hline $\mathrm{C}_{2} \mathrm{H}_{3}$ & $3,22 \pm 0,1$ & - & $(19)$ \\
\hline $\mathrm{C}_{2} \mathrm{H}_{4}$ & $2,73 \pm 0,1$ & - & $(19)$ \\
\hline
\end{tabular}

$\mathrm{D}(\mathrm{HC}-\mathrm{CH})=9 ; 88 \mathrm{eV}(\mathrm{a})$ et $\mathrm{D}\left(\mathrm{HC}_{2}-\mathrm{H}\right)=4,90 \mathrm{eV}(\mathrm{b})$

\section{BIBLIOGRAPHIE}

[1] Locht, R., Momigny, J., Int. J. Mass Specîr, Ion Phys., 2, 425 (1969).

[2] Locht, R., MomignY, J., Int. J. Mass Spectr. Ion. Phys., à paraître (1970).

[3] Kraus, K., Dissertation, Hamburg (1961).

[4] Schulz, G. J., Phys. Rev., 128, 178 (1962).

[5] Sмith, L. G., Phys. Rev., 51, 263 (1937).

[6] Von TREPKA, L., Z. fur Naturf., 18a, 1295 (1963).

[7] Dibeler, V. H., Krauss, K., Reese, R. M., Harlee, F. N., J. Chem. Phys., 42, 3791 (1965).

[8] HerZBerg, G., Molecular Spectra and Molecular Structure. III. Electronic Spectra of Diatomic Molecules (Ed. D. Van Nostrand, 
Published in: Bulletin de la Classe des Sciences. Académie Royale de Belgique (1970), vol. LVI, pp. 788-817.

Status: Postprint (Author's Version)

New-York, 1967).

[9] HerzBerg, G., Molecular Spectra and Molecular Structure. II. Spectra of Diatomic Molecules (Ed. Van Nostrand, New York, 1967, $2^{\mathrm{e}}$ édition).

[10] Smith, S. J., Burch, D. S., Phys. Rev., 116, 1125 (1959).

[11] Vedeneyev, V. I,. Gurvich, L. V., Condrat'yev, V. N., Frankevich, Y. E., Bond Energies, Ionization Potentials, Electronaffinities (ed. Arnold, 1966).

[12] Cade, P. E., Proc. Phys. Soc, 91, 842 (1967)

[13] Locht, R., Momigny, J., Chem. Phys. Lett., à paraître (1970).

[14] HONIG, R. E., J. Chem. Phys., 22, 126 (1954).

[15] Dowell, J. T., Scharp, T. E., Phys. Rev., 167, 124 (1968).

[16] Ballik, E. A., Ramsay, D. A., Astrophys. J., 137, 84 (1963).

[17] Drowart, J., Burns, R. P., DeMaria, G., Inghram, M. G., J. Chem. Phys., 31, 1131 (1959).

[18] Maslov, P. G., Khim. i. Techn. Topliv i. Masel 3, 10, 50 (1958); Chem. Abstr., 53, 1911a (1959)

[19] Benson, S. W, J. Chem. Educ, 42, 502 (1965).

[20] Chupka, W. A., Lifshrtz, C, J. Chem. Phys., 48, 1109 (1968).

[22] Seman, M. L., Branscomb, L. M., Phys. Rev., 125, 1602 (1962).

[23] Berry, R. S., Reimann, C. W., J. Chem. Phys., 38, 1540 (1963). 